\title{
WALL COPS AND ROBBERS
}

by

Fionn Mc Inerney, BSc in Mathematics and Its Applications, Ryerson

University, 2013

A thesis presented to Ryerson University

in partial fulfillment of the

requirements for the degree of

Master of Science

in the Program of

Applied Mathematics

Toronto, Ontario, Canada, 2015

(C) Fionn Mc Inerney 2015 


\section{AUTHOR'S DECLARATION FOR ELECTRONIC SUBMISSION OF A THESIS}

I hereby declare that I am the sole author of this thesis. This is a true copy of the thesis, including any required final revisions, as accepted by my examiners.

I authorize Ryerson University to lend this thesis to other institutions or individuals for the purpose of scholarly research.

I further authorize Ryerson University to reproduce this thesis by photocopying or by other means, in total or in part, at the request of other institutions or individuals for the purpose of scholarly research.

I understand that my thesis may be made electronically available to the public. 


\author{
Abstract \\ Wall Cops and Robbers \\ Master of Science (MSc) 2015
}

Fionn Mc Inerney

Applied Mathematics

Ryerson University

Wall Cops and Robbers is a new turn-based game played on graphs. It is inspired by the games of Cops and Robbers and the Angel Problem. The objective of the game is for the cops to capture the robber by surrounding him with walls. The wall capture time of a graph $G$, written $W_{c_{t}}(G)$, is the least number of moves it takes for one cop to capture the robber in $G$. The wall cop number of a graph $G$, written $W_{c}(G)$, is the least number of cops it takes to capture the robber in $G$. The thesis explores these two parameters for various graph classes including trees, hypercubes, grids, and tilings of the plane. We also introduce a variant of the game called Wall Cops and Wall Robbers. In this game, the robber creates a wall on visiting a vertex, disallowing a move to a previously visited vertex. 


\section{Acknowledgements}

I would like to thank the faculty and staff in the Department of Mathematics at Ryerson University for their support in helping me accomplish everything I have done in both my undergraduate and graduate degrees at Ryerson University. In particular, I would like to thank Dr. Peter Danziger and Dr. Dejan Delić for being part of my thesis committee. Also, a special thanks to Dr. Delić for hiring me as a summer undergraduate researcher and introducing me to research in graph theory. I would also like to thank Dr. Jean-Paul Pascal for chairing my defense.

I am most thankful to my supervisor for this thesis, Dr. Anthony Bonato, for his continuous support throughout the process. He gave me a lot of confidence by proposing to do a thesis primarily based on original results because he thought I was capable. That meant a lot to me and it not only helped me with my graduate studies but it will also help me in the future.

Last but not least, I would like to thank my family and friends for their support. My parents, Sean and Pascale, and my brother Killian have all been encouraging and helpful throughout my life. 


\section{Contents}

List of Figures vii

Chapter 1. Introduction 1

1.1. Introduction to Wall Capture Time and Wall Cop Number 1

1.2. Introduction to Graph Theory 5

1.3. Outline of Thesis 10

$\begin{array}{lll}\text { Chapter 2. Wall Capture Time } & 13\end{array}$

2.1. Introduction 13

2.2. Wall Capture Time of a Subgraph 16

2.3. Wall Capture Time of a 3-regular Tree 17

2.4. Wall Capture Time of a $k$-regular Tree 19

2.5. Wall Capture Time of a Hypercube 22

$\begin{array}{lll}\text { Chapter 3. Grids } & 25\end{array}$

3.1. Wall Capture Time of an Infinite Hexagonal Grid 25

3.2. Wall Capture Time of an Infinite Cartesian Grid 35

3.3. Wall Capture Time of an Infinite Triangular Grid 58

3.4. Wall Capture Time of an Infinite Strong Grid 64

3.5. Wall Capture Time of an n-layered Infinite Cartesian Grid 74 
Chapter 4. Wall Cop Number

4.1. Introduction

4.2. Wall Cop Number of an Infinite-layered Infinite Hexagonal grid

4.3. Wall Cop Number of an Infinite-layered Infinite Cartesian grid

4.4. Wall Cop Number of Layered Strong Grids

Chapter 5. Wall Cops and Wall Robbers 95

5.1. Introduction 95

5.2. Wall Robber Capture Time of an Infinite Hexagonal Grid 97

5.3. Wall Robber Capture Time of an Infinite Cartesian Grid 99

$\begin{array}{lll}\text { Chapter 6. Conclusion and Open Problems } & 107\end{array}$

6.1. Open Problems 108

$\begin{array}{ll}\text { Bibliography } & 111\end{array}$ 


\section{List of Figures}

1.1 A labelled graph $G$.

1.2 An example of a finite graph. The vertices of this graph are 1, 2, 3 and 4 . The edges are 12, 23, 34, and 41. $N(2)=\{1,3\}$. The graph is 2-regular since all of its vertices have degree 2. The cop number of this graph is 2 .

$\begin{array}{lll}1.3 & \text { Figure } 1.2 \text { is a subgraph of this graph. } & 6\end{array}$

1.4 An infinite one-way path. 6

1.5 A path of order 5 and length 4 denoted by $P_{5}$. 7

$1.6 C_{5}$, a cycle of order 5 . 8

1.7 A few examples of hypercubes. 8

1.8 The Cartesian product of two paths of order 4 (left), written $P_{4} \square P_{4}$. The strong product of two paths of order 4 (right), written $P_{4} \otimes P_{4}$.

2.1 A star with 5 vertices, written $K_{1,4}$. 14

2.2 A 3-regular tree of height 2. 14

2.3 A cube, written $Q_{3}$, with labelled vertices. 16 
3.1 An infinite hexagonal grid with labelled coordinates.

3.2 Cop and robber's moves in Case A. The labels correspond to the player's turn number and location on the closest vertex up and to the right. When both a cop label and a robber label are needed, the cop label appears below the robber label. The notation $R_{4,5}$ means that the robber's $4^{\text {th }}$ and $5^{\text {th }}$ move are on that vertex. The cop's first move $C_{1}$ is not shown in the figure as it is sufficiently far away from these vertices.

3.3 Cop and robber's moves in Case B. The labels correspond to the player's turn number and location on the closest vertex up and to the right. When both a cop label and a robber label are needed, the cop label appears below the robber label. The cop's first move $C_{1}$ is not shown in the figure as it is sufficiently far away from these vertices.

3.4 The two possible traps the cop can build. The two walls of the trap are in black and the middle vertex is in grey.

3.5 Cop and robber's moves in Case 1.1.1. The labels correspond to the player's turn number and location on the closest vertex up and to the right. When both a cop label and a robber label are needed, the cop label appears below the robber label. $R_{8,9}$ means that the robber's $8^{\text {th }}$ and $9^{\text {th }}$ moves are on that vertex. 
The cop's first move $C_{1}$ is not shown in the figure as it is sufficiently far away from these vertices.

3.6 Cop and robber's moves in Case 1.1.2.

3.7 Cop and robber's moves in Case 2.

3.8 An infinite triangular grid. Vertices are omitted.

3.9 The first 10 moves of the cop shown as black vertices, with the first move not shown as it is far away from the hexagon. Also, the assumption here for the tenth move is that the robber moved up towards the TLC or TRC.

3.10 The parallelogram that is built with the $5 \times 4$ parallelogram built inside it with the robber's starting vertex labelled $R$ and the cop's first move labelled $\mathrm{C}$ and the rest of his moves to construct the parallelogram not labelled. This figure assumes the robber moves in a way that allows the sides of the parallelogram to be parallel to the sides of the hexagon of length three.

3.11 Corners of the strong grid with the diagonal edges omitted.

3.12 Cop's strategy to stop robber reaching a side from a distance of five moves. In each of the figures, $a, b$, and $c$ represent the fourth wall the cop would build if the robber moved diagonally up and to the left or straight up or diagonally up and to the 
right, respectively. The final wall to build from there is obvious then.

$3.1312 \times 12$ box robber is trapped inside with the $6 \times 6$ box and $4 \times 4$ cross inside.

3.14 Representation with compressed dimensions of the $(8 n+3) \times$ $(7 n+2)$ box built on one of the $n$ levels. The grey vertices represent the corners built at the beginning.

3.15 Smaller representation of $7 n \times 4$ box built on one of the $n$ levels with the $4 \times 4$ box built inside it.

4.1 An infinite 4-regular tree.

4.2 Cops and robber's moves in $H_{\infty_{\infty}}$. The labels correspond to the player's turn number and location on the closest vertex up and to the right. When both a cop label and a robber label are needed, the cop label appears below the robber label. $R_{4,10,11}$ means that the robber's $4^{t h}, 10^{t h}$, and $11^{\text {th }}$ moves are on that vertex. The cops' first move $C_{1}$ is not shown in the figure as it is sufficiently far away from these vertices.

4.3 Fifth layer of the cube ( $x y$ plane at $z=5$ ). Cops' first moves are represented by the four vertices in the corners. The figure is the same for the $z=-5$ layer of the cube.

4.4 Robber's position in red after $2^{\text {nd }}$ turn for cops in blue. 
4.5 Robber's position in red after $3^{\text {rd }}$ turn for cops in green.

4.6 Robber's position in red after $3^{\text {rd }}$ turn for cops in green.

4.7 Robber's position in red before $4^{\text {th }}$ turn for cops.

4.8 Robber's position in red at $(0,-1,0)$ (cannot be seen) after $4^{\text {th }}$ turn for cops in yellow.

4.9 Robber's position in red at $(0,-1,0)$ (cannot be seen) after $4^{\text {th }}$ turn for cops in yellow.

4.10 Robber's position in red at $(0,-1,0)$ (cannot be seen) after $4^{\text {th }}$ turn for cops in yellow.

4.11 Robber's position in red before $5^{t h}$ turn for cops.

4.12 Robber's position in red (cannot be seen) after $5^{\text {th }}$ turn for cops in magenta.

4.13 Robber's position in red (cannot be seen) after $5^{\text {th }}$ turn for cops in magenta.

5.1 Cop and robber's moves. The labels correspond to the player's turn number and location on the closest vertex up and to the right. The cop's first move $C_{1}$ is not shown in the figure as it is sufficiently far away from these vertices.

5.2 Cop and robber's moves in Case 1.1. The labels correspond to the player's turn number and location on the closest vertex up and to the right. The cop's first move $C_{1}$ is not shown in the 
figure as it is sufficiently far away from these vertices. The cop's tenth move $C_{10}$ is not shown in the figure as it is irrelevant in the capture of the robber.

5.3 Cop and robber's moves in Case 1.2. The labels correspond to the player's turn number and location on the closest vertex up and to the right. The cop's first move $C_{1}$ is not shown in the figure as it is sufficiently far away from these vertices. The cop's tenth move $C_{8}$ is not shown in the figure as it is irrelevant in the capture of the robber.

5.4 Cop and robber's moves in Case 2. The labels correspond to the player's turn number and location on the closest vertex up and to the right. The cop's first move $C_{1}$ is not shown in the figure as it is sufficiently far away from these vertices. The cop's tenth move $C_{9}$ is not shown in the figure as it is irrelevant in the capture of the robber. 
CHAPTER 1

\section{Introduction}

\subsection{Introduction to Wall Capture Time and Wall Cop Number}

Wall Cops and Robbers is a new turn-based game played on graphs. There are two players: a cop and a robber. The game starts with the cop building a "wall" on a vertex which blocks off that vertex so that the robber cannot occupy it. After that, the robber selects a vertex. The cop can build a wall on any vertex on his turn except for the vertex that the robber currently occupies. Hence, we may think of the cop as playing off the graph. The robber, however, can only move along an edge to an adjacent vertex each turn. The robber is also allowed to skip his turn, but he may not move back to the vertex he occupied on his previous turn. Both the cop and the robber have full knowledge of all the moves that have taken place. The objective of the game for the cop is to build walls to block off all adjacent vertices to the robber so that the robber can no longer move on his next turn. The objective of the game for the robber is to evade capture by the cop for as long as possible.

The wall capture time, written $W_{c_{t}}$, of a graph is the least number of moves it takes for the cop to capture the robber on the given graph given 
that the cop and robber have both played their best strategies. The wall cop number of a graph, written $W_{c}$, is the least number of cops it takes for the cops to capture the robber on a given graph. The idea for Wall Cops and Robbers is inspired by the games of Cops and Robbers and the Angel Problem.

As a simple example, consider the game played on the graph $G$ in Figure 1.1. We label the vertices 1, 2, 3, 4, 5, and 6 as in Figure 1.1 and the cop builds a wall on vertex 3 . If the robber chooses 1 or 2 , then he will be stuck in the left triangle and will lose on the next turn. Any of the vertices in the right triangle would be a good choice for the robber, so he chooses 5 . The cop builds a wall on 4 . The robber can either move to 6 or skip his turn and remain at 5 ; since both give the same result of him losing next turn, let us say he moves to 6 . The cop builds a wall on 5 and captures the robber as he can no longer move. Since it takes exactly three moves for the cop to capture the robber with both sides playing at their best, we have that $W_{c_{t}}(G)=3$. Also, since it only took one cop to capture the robber, we have that $W_{c}(G)=1$. 


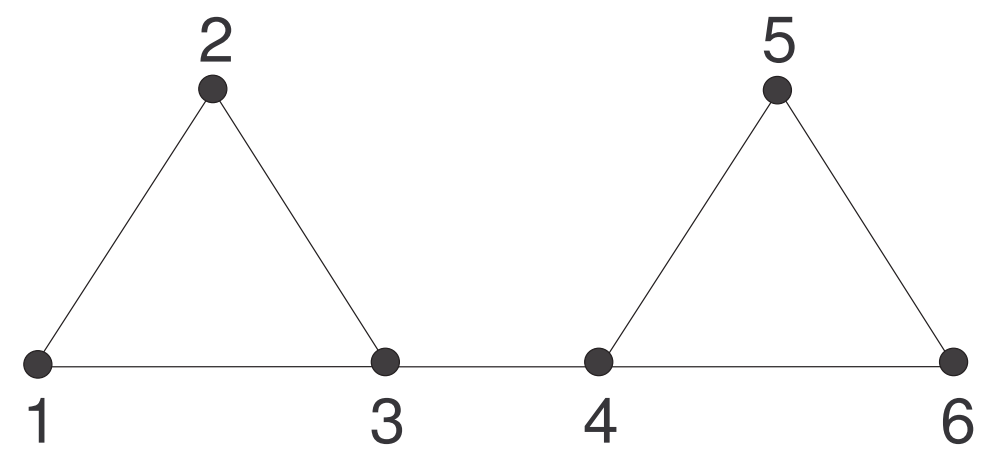

Figure 1.1. A labelled graph $G$.

The game of Cops and Robbers was first introduced by Quilliot in his Ph.D. Thesis [10] in 1978 and independently by Nowakowski and Winkler in [9] in 1983. Cops and Robbers has all the same rules as Wall Cops and Robbers except for the following. In Cops and Robbers, instead of the cop building walls, the cop actually moves the same as the robber and the cop wins if he lands on the robber. For more information on Cops and Robbers, see $[\mathbf{1}],[\mathbf{3}]$, and [7].

The Angel Problem was first introduced by John H. Conway in [2] in 1982. The Angel Problem has similarities and differences with Wall Cops and Robbers. The Angel Problem is a turn-based game played on either an infinite chessboard or an infinite 3-dimensional chessboard. The game is played by an Angel and the Devil which we call robber and cop, respectively, in Wall Cops and Robbers. The Angel has power $k$, where $k$ is a positive integer, which allows the Angel to move $k$ spaces in any direction. We can think of this as the Angel having $k$ moves on his turn 
where he can only move to an adjacent space on each move. In Wall Cops and Robbers, the robber is the equivalent of an Angel of power $k=1$. The Devil has the same power as the cop in Wall Cops and Robbers, except that he eats squares in the Angel Problem while the cop builds walls on vertices. The objective of the game is the exact same as the objective of Wall Cops and Robbers except in this game it is the Angel trying to elude capture by the Devil on a chessboard which has spaces instead of a graph with vertices but a chessboard can be represented by a graph with vertices. The Angel Problem is only concerned with who wins and not how many moves it takes for the Devil to win. For more information on the Angel Problem, see [5]. Wall Cops and Robbers takes the rules from the Angel Problem of how the Angel and Devil play and combines them with the playing surfaces of Cops and Robbers so that the game can be played on many different graphs instead of just a chessboard. Also, the parameters from Cops and Robbers known as cop number and capture time are adjusted so that they can be applied to Wall Cops and Robbers. These parameters will be defined further on in the chapter.

We also mention Firefighting on graphs which has some similar aspects to our game. The Firefighter Problem was introduced by Hartnell in 1995 at [8]. Firefighting on graphs is a turn-based game where firefighters try to contain a fire. The fire starts at some vertex and then the firefighters 
place themselves on vertices making these vertices fire-proof. Then on the next turn the fire spreads to all adjacent vertices that are not fireproof and the firefighters move to new vertices with the previous vertices always remaining fire-proof. The game continues like this and one of the goals for the firefighters may be to contain the fire so that it spreads no more and save as many vertices from the fire as possible. See the survey [6] for various desired outcomes of the game.

\subsection{Introduction to Graph Theory}

The game of Wall Cops and Robbers is played on graphs and thus, we will introduce some of the terminology from Graph Theory in this section. A graph $G$, consists of a set of vertices represented by dots denoted by $V(G)$ and a set of edges represented by lines denoted by $E(G)$. The edges connect the vertices and when two vertices are connected by an edge, we say that these vertices are adjacent. An edge between two vertices $u$ and $v$ would be written $u v$. All graphs considered throughout the thesis are undirected which means the edges do not have any direction. The order of $G$ is denoted by $|V(G)|$ which is the total number of vertices in $G$. The degree of a vertex is equal to the number of vertices that are adjacent to it. The neighbour set of a vertex $u$ is denoted by $N(u)$ which consists of all vertices that are adjacent to $u$. A graph is said to be $k$-regular if each vertex has degree $k$. A subgraph $H$ of a graph $G$ is a graph that consists of a vertex set denoted by $V(H)$ and an edge set denoted by $E(H)$ such 
that $V(H)$ is a subset of $V(G)$ and $E(H)$ is a subset of $E(G)$. A graph is infinite if $|V(G)|=\infty$, that is there are an infinite number of vertices, otherwise a graph is finite.

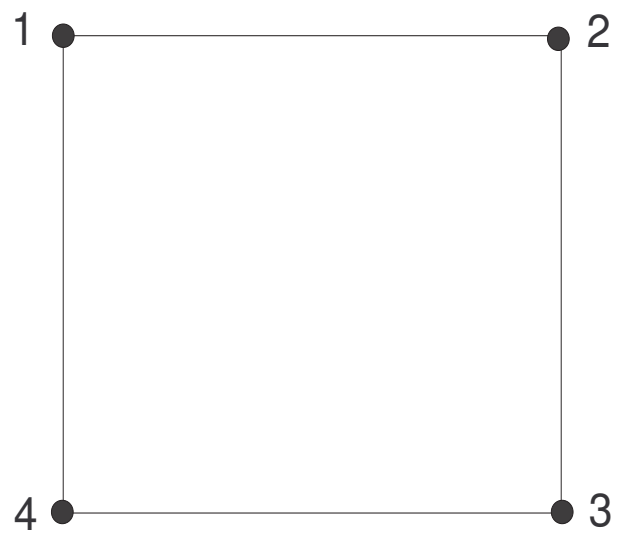

Figure 1.2. An example of a finite graph. The vertices of this graph are 1, 2, 3 and 4 . The edges are 12,23,34, and 41. $N(2)=\{1,3\}$. The graph is 2-regular since all of its vertices have degree 2 . The cop number of this graph is 2 .

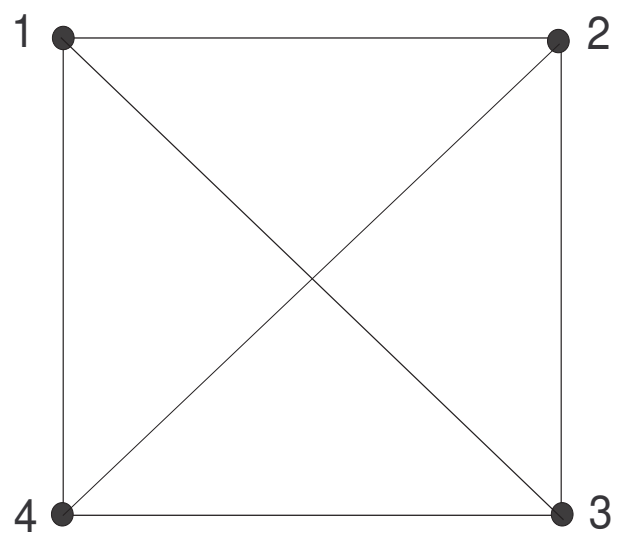

FIGURE 1.3. Figure 1.2 is a subgraph of this graph.

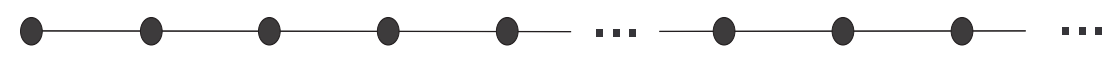

Figure 1.4. An infinite one-way path. 
The cop number of a graph $G$, denoted by $c(G)$, is the least number of cops it takes to capture the robber given that the cops and robber use the best strategies possible in $G$. The $k$-capture time of a graph $G$ denoted by $\operatorname{capt}_{k}(G)$ is the least number of turns it takes for $k$ cops to capture the robber in $G$ (not including the initial placement turn).

A path of order $n$ denoted by $P_{n}$, is a sequence of $n$ vertices such that each vertex is adjacent to the next vertex in the sequence. The number of edges in a path is called the length of the path. The distance between two vertices $u$ and $v$ is the length of the shortest path connecting these vertices or infinity otherwise. A graph is said to be connected if there exists a path between any two vertices; otherwise the graph is disconnected. A cycle of order $n$ represented by $C_{n}$, is a path of order $n$ in which there exists an edge between the first and last vertex in the sequence. A tree is any connected graph that contains no cycles. A hypercube of dimension $n$ denoted by $Q_{n}$, has vertices elements of $\{0,1\}^{n}$ with two vertices being adjacent if they differ in exactly one coordinate. A hypercube of dimension $n$ can also be obtained by adding edges between corresponding vertices between two copies of $Q_{n-1}$ with $Q_{0}$ being a single vertex.

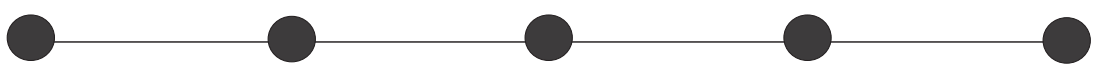

Figure 1.5. A path of order 5 and length 4 denoted by $P_{5}$. 


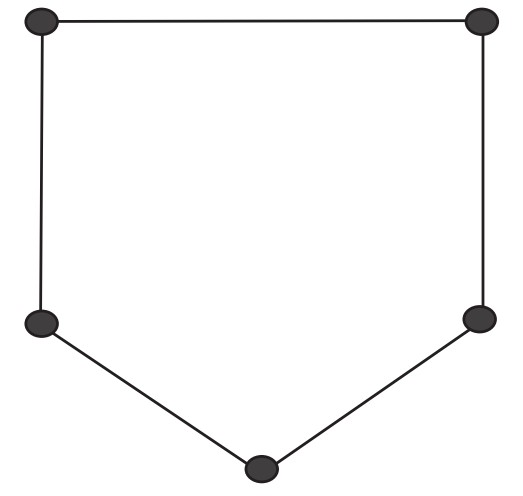

Figure 1.6. $C_{5}$, a cycle of order 5 .

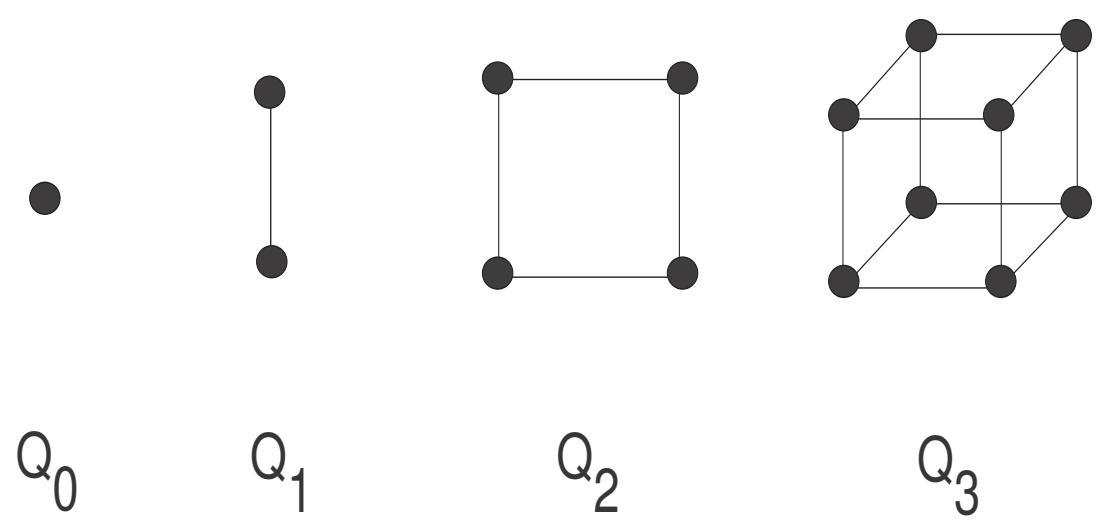

Figure 1.7. A few examples of hypercubes.

A graph product is an operation done on two graphs to produce a new graph. There exist many different graph products but we will focus on the Cartesian and strong products. First, we will introduce the Cartesian product of two sets. The Cartesian product of two sets $A$ and $B$ is denoted by $A \times B$. For example, if $A=\{1,2,3\}$ and $B=\{4,5\}$, then $A \times B=\{(1,4),(1,5),(2,4),(2,5),(3,4),(3,5)\}$. The Cartesian product of two graphs $G$ and $H$, denoted by $G \square H$, consists of the vertex set $V(G) \times V(H)$ and the edge set is obtained as follows: any two vertices 
$(a, b)$ and $(c, d)$ are adjacent in $G \square H$ if $a=c$ and $b$ and $d$ are adjacent in $H$ or $b=d$ and $a$ and $c$ are adjacent in $G$. The Cartesian product is commutative in that $G \square H \cong H \square G$.

The strong product of two graphs $G$ and $H$, denoted by $G \otimes H$, consists of the vertex set $V(G) \times V(H)$ and the edge set is obtained as follows: any two vertices $(a, b)$ and $(c, d)$ are adjacent in $G \otimes H$ if $a=c$ and $b$ and $d$ are adjacent in $H$ or $b=d$ and $a$ and $c$ are adjacent in $G$ or $a$ and $c$ are adjacent in $G$ and $b$ and $d$ are adjacent in $H$. The strong product is also commutative. It is important to notice that the first two conditions of adjacency in the strong product are the same as in the Cartesian product but the strong product also has a third condition making it possible to have more edges than the Cartesian product. Further, they both have the same vertex set and thus, the Cartesian product of two graphs is a subgraph of the strong product of these graphs.
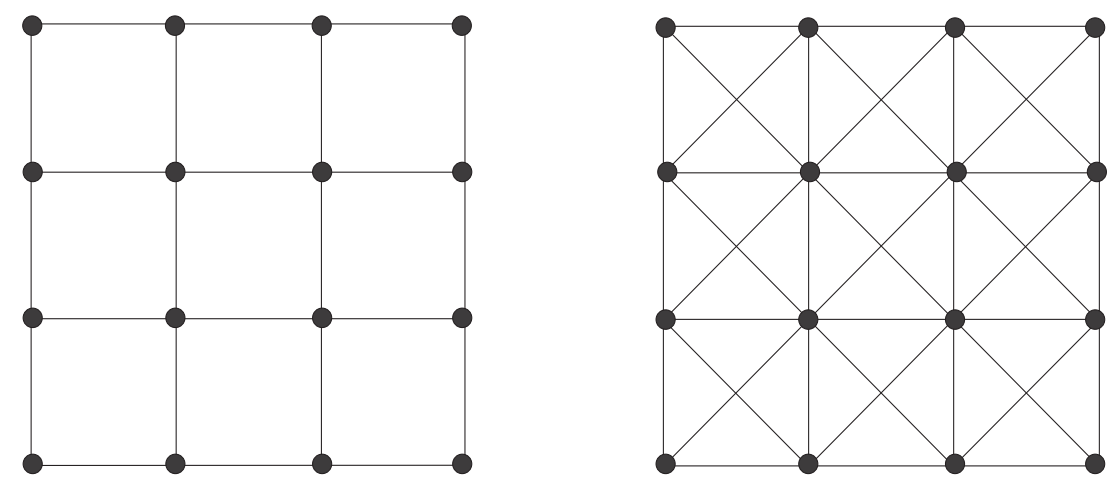

Figure 1.8. The Cartesian product of two paths of order 4 (left), written $P_{4} \square P_{4}$. The strong product of two paths of order 4 (right), written $P_{4} \otimes P_{4}$. 


\subsection{Outline of Thesis}

In our introductory chapter, we introduced the new game of Wall Cops and Robbers, along with associated parameters $W_{c_{t}}$ and $W_{c}$. We also introduced graphs and graph theoretic terminology. In Chapter 2, we introduce wall capture time more thoroughly with a few small examples. We discuss and prove the monotonicity of $W_{c_{t}}$ in terms of subgraphs. Also, we give results and proofs of wall capture time for hypercubes and certain trees. In Chapter 3, we give results and proofs of wall capture time for grids in 2 and 3-dimensions and tilings of the plane. We look at grids such as the Cartesian product of two infinite paths, written $P_{\infty} \square P_{\infty}$, the strong product of two infinite paths, written $P_{\infty} \otimes P_{\infty}$, and tilings such as hexagonal and triangular tilings. In Chapter 4, we introduce wall cop number more thoroughly. We discuss and prove monotonicity of $W_{c}$ in terms of subgraphs. In addition, we give results and proofs of $W_{c}$ for 3 -dimensional grids. In Chapter 5 , we introduce a variation of the game of Wall Cops and Robbers and give some results and proofs in that variation. Finally, in Chapter 6, we give a short summary of the results followed by some open problems.

We highlight that the majority of the results for $W_{c_{t}}$ and $W_{c}$ are upper bounds as good lower bounds can be difficult to find and prove, therefore, making it hard to prove equality in many of the cases. The lower bounds are difficult to prove as they require proving that the cop 
or cops are using their best strategy possible while simultaneously the robber is using his best strategy possible. An upper bound, in contrast, is more readily proven. In this case, we need to provide a strategy for the cop or cops such that even with the robber using his best strategy, the cop or cops can win in at most that many turns. 



\section{CHAPTER 2}

\section{Wall Capture Time}

\subsection{Introduction}

The game of Wall Cops and Robbers has the cops try to capture the robber while the robber tries to evade capture forever. The most basic aspect of the game is which side wins on a given graph. Note that the cop is guaranteed to win on any finite graph since eventually, all the vertices except the last one the robber occupies will be covered with a wall. Therefore, a more interesting parameter to look at is how fast the cop can capture the robber on a given graph which is known as wall capture time.

Recall that the wall capture time of a graph $G$, written $W_{c_{t}}(G)$, is the least number of moves it takes for the cop to capture the robber in $G$ given that the cop and robber have both played their best strategies.

We will first look at a few examples of wall capture time for some small graphs. We begin the discussion by considering a star with 5 vertices, written $K_{1,4}$, as seen in Figure 2.1 below. The cop plays on the central vertex of degree 4. No matter where the robber places himself, he is captured and therefore, $W_{c_{t}}\left(K_{1,4}\right)=1$. In fact, for any star with $k+1$ vertices, written $K_{1, k}$, we also have that $W_{c_{t}}\left(K_{1, k}\right)=1$. 


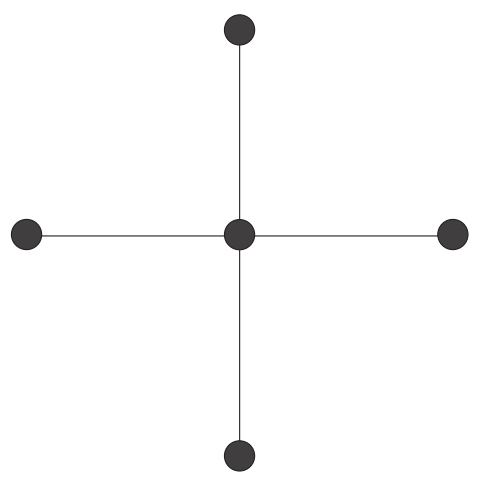

Figure 2.1. A star with 5 vertices, written $K_{1,4}$.

Now we consider a 3-regular tree of height 2 as seen in Figure 2.2 below. The cop plays on the root vertex which is the topmost vertex. The robber places himself on any vertex of the second level of the tree, with the first level of the tree being just the root vertex, otherwise he is captured next turn. The cop plays on one of the leaves adjacent to the robber. The robber moves to the other leaf or skips his turn. The cop moves to the vertex adjacent to the robber that does not have a wall and the robber is captured in a total of 3 moves. Therefore, the wall capture time of a 3-regular tree of height 2 is 3.

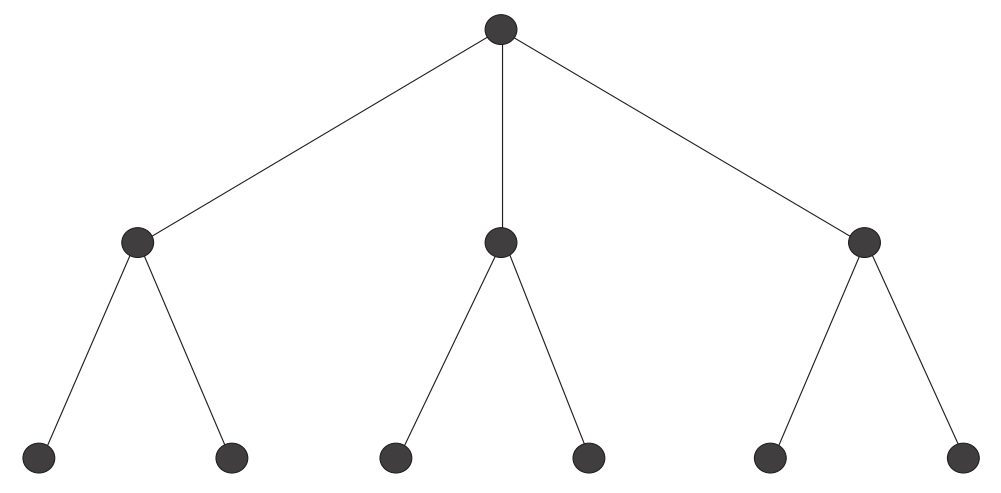

Figure 2.2. A 3-regular tree of height 2. 
As a last example, let us consider the wall capture time of the cube, written $Q_{3}$, as in the figure below. The cop will build a wall on 1 . If the robber chooses any vertex other than 7 , then he will be captured in 5 moves. If the robber chooses 2,3 or 4 , then the cop will keep the robber confined to the top level of the cube and then capture him on this level for 5 moves. If the robber chooses 5 , then the cop will play on 8 which forces the robber to move to 6 . The cop would play on 2 . If the robber skips his turn, then the cop plays on 7 and then adjacent to the robber the next turn. If the robber moves to 7 , then the cop plays on 3 and then on 6 the next turn since the robber cannot move back there since he occupied it last turn. Thus, the robber chooses 7 as then he will be captured in 6 moves total. The cop will play adjacent to the robber and then the robber will skip his turn. The cop will play adjacent to the robber again and then the robber will move to the open adjacent vertex and skip his turn for the remainder of the game resulting in 6 moves for the cop. If the cop did not play adjacent to the robber both times, then the robber would simply skip his turn until only one of his neighbours is open and at that point he would move to that vertex and skip his turn for the remainder of the game. Therefore, the wall capture time of $Q_{3}$ is 6 or $W_{c_{t}}\left(Q_{3}\right)=6$. 


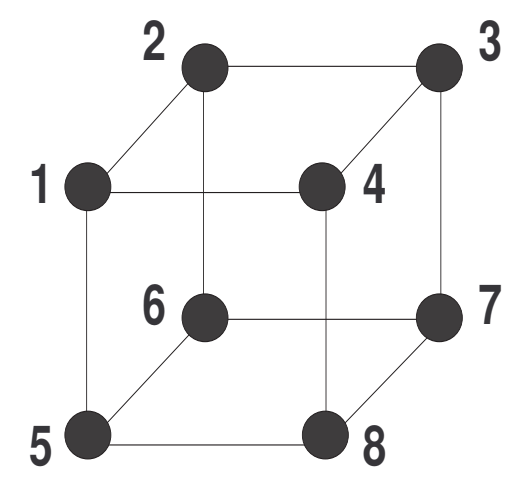

Figure 2.3. A cube, written $Q_{3}$, with labelled vertices.

\subsection{Wall Capture Time of a Subgraph}

Wall capture time is monotonic relative to subgraphs. This result gives lower bounds for the wall capture time of graphs if the wall capture time of one of their subgraphs is known. Also, if the wall capture time of a graph is known then this result gives upper bounds for the wall capture time of subgraphs of that graph.

TheOREM 2.2.1. $W_{c_{t}}(H) \leq W_{c_{t}}(G)$ if $H$ is a subgraph of $G$.

Proof. The cop plays two games simultaneously: one in $G$ using his winning strategy there, and one in $H$. The robber is confined to $H$, and we view moves in $H$ by the robber as moves in $G$. The cop adapts his strategy in $G$ to one in $H$ as follows. If the cop builds a wall in $G$ on a vertex $u$ also in $H$, then he builds a wall on $u$ in the game on $H$. Each time the cop builds a wall in $V(G) \backslash V(H)$, he can just choose any vertex without a wall in $H$, and build a wall there. 
The adapted strategy in $H$ is winning for the cop, as in each round, the vertices without walls available to the robber is a subset of vertices without walls in $G$. Hence, the robber will be captured in $W_{c_{t}}(G)$-moves or fewer in $H$.

To show the bound is sharp, take the example of a path of length 20 . The wall capture time of this path is 4 . The path $P_{19}$ is a subgraph of $P_{20}$ but also has wall capture time of 4 .

\subsection{Wall Capture Time of a 3-regular Tree}

The height of a tree $T$, written $h(T)$, is the length of the longest path from the root vertex to a leaf vertex. Figure 2.2 is an example of a $3-$ regular tree of height 2. A 3-regular tree, written $T$, is a tree in which every vertex has degree 3 except the leaves.

The wall capture time of a 3-regular tree of height 0 is 1 since that is just a graph with one vertex. The wall capture time of a 3-regular tree of height 1 is 2 as the cop would play on the root vertex forcing the robber to play on a vertex of degree one. The cop would then play on any other vertex and win. The wall capture time of a 3-regular tree of height 2 is 3 as was shown in an earlier example.

TheOREM 2.3.1. $W_{c_{t}}(T)=h(T)+2$ for finite 3-regular trees $T$ with $h(T) \geq 3$. 
Proof. The cop plays on the root vertex. The robber then places himself on the second level of the tree; otherwise, if he goes further down, the cop will move to the vertex on the level above him, reducing the wall capture time. If the cop ever moves two or more levels below the robber, then the robber will move down whichever branch the cop did not place himself on, therefore, without loss of generality, the cop then moves to a vertex adjacent to the robber on the level below him. The robber moves down to the third level. The cop moves to a vertex adjacent to the robber on the level below him. The cop and robber will keep moving like this until the robber gets to the second last level of the tree then the cop will move to the vertex adjacent to the robber on the level above him.

If the robber ever skipped his turn before reaching the second last level, then the cop would play adjacent to the robber on the level below him, forcing the robber to move back up the tree or skip his turn if he cannot. If the robber can move back up the tree, he will get two more moves out of the cop and if he cannot then he will get only one more move out of the cop. Hence, the robber would never skip his turn before reaching the second last level.

Now, the robber will stay still on the second last level of the tree since otherwise he has to move to a leaf vertex and will be captured next turn. The cop will move to one of the leaf vertices adjacent to the robber. 
The robber moves to the other leaf or stays still. The cop moves to the vertex adjacent to the robber that does not have a wall and the robber is captured in a total of the height of the tree +2 moves.

We have equality for the wall capture time as the cop cannot improve upon this strategy. We have shown that if he plays further down the tree than just one level below the robber, then it makes no difference as the robber will just move down the other branch. If the cop plays on the level above the robber at any point other than when the robber reaches the second last level of the tree, then the robber will just skip his turn and gain one extra move out of the cop. Therefore, the cop's best strategy is as above and we have that $W_{c_{t}}(T)=h(T)+2$ for $h(T) \geq 3$.

\subsection{Wall Capture Time of a $k$-regular Tree}

A $k$-regular tree is a tree $T_{k}$, in which every vertex has degree $k$ except the leaves.

The wall capture time of a $k$-regular tree of height 0 is 1 and of height 1 is 2 using the same proofs as with 3-regular trees.

THEOREM 2.4.1. The wall capture time of a $k$-regular tree of height 2 is $k$.

Proof. The cop plays on the root vertex. The robber places himself on the second level of the tree otherwise he is captured next turn. The cop plays on one of the leaves adjacent to the robber. The robber stays still 
until the cop has built a wall on all adjacent vertices and thus, captures the robber since moving to a leaf vertex results in being captured on the next turn for the robber. That vertex the robber stays on for the duration of the game has degree $k$. Therefore, the wall capture time of a $k$-regular tree of height 2 is $k$.

TheOREM 2.4.2. $W_{c_{t}}\left(T_{k}\right)=(k-2) h\left(T_{k}\right)-k+5$ for finite $k$-regular trees with $h\left(T_{k}\right) \geq 3$ and $k \geq 3$.

Proof. The cop plays on the root vertex. The robber then places himself on the second level of the tree; otherwise, if he goes further down, the cop will move to the vertex on the level above him, reducing the wall capture time. If the cop ever moves two or more levels below the robber, then the robber will treat it as the cop moving only one level below him on that same branch, therefore, without loss of generality, the cop then moves to a vertex adjacent to the robber on the level below him. The robber will skip his turn until only one option remains for him to move down at which point he will move down since if he moves down earlier then the cop will move to the vertex on the level above him. The robber skips his turn $k-3$ times so that only one option remains for him to move down as the cop builds $k-3$ more walls on the adjacent vertices on the level below the robber. The robber moves down to the third level. The cop moves to a vertex adjacent to the robber on the level below him. The robber skips his turn $k-3$ times while the cop builds $k-3$ more 
walls on the adjacent vertices on the level below the robber. The cop and robber will keep moving like this until the robber gets to the second last level of the tree then the cop will move to the vertex adjacent to the robber on the level above him.

If the robber ever backtracked when able to in any of the previous moves, then the robber would be captured in three moves as the cop would move to the adjacent vertex below him, then the robber would move further up, then the cop would move to the adjacent vertex above, then the robber will be forced to skip his turn and the cop will capture him on the next turn.

If the robber ever skipped his turn $k-2$ times on one level on any level other than the second last level, then the cop would play adjacent to the robber on the level below him which would force the robber to move back up the tree and be captured in at most two moves as in the 3-regular finite tree proof.

Now, the robber will stay still on the second last level of the tree since otherwise he has to move to a leaf vertex and will be captured next turn. The cop has to make $k-1$ moves to capture the robber now. The cop therefore has made one move placing himself on the root vertex. Then $(k-2)\left(h\left(T_{k}\right)-2\right)$ moves for each level of the tree that is not the top or bottom two levels since the robber skips his turn $k-3$ times at each of these levels. Finally, once the robber reaches the second last level of the 
tree, the cop makes one move above the robber and $k-1$ moves below the robber to capture him resulting in a total of $(k-2)\left(h\left(T_{k}\right)-2\right)+k+1$ moves. By multiplying, we have a total of $(k-2) h\left(T_{k}\right)-k+5$ moves.

We have equality for the wall capture time as the cop cannot improve on the strategy above for the same reasons as in a finite 3-regular tree. Therefore, the cop's best strategy is as above and $W_{c_{t}}\left(T_{k}\right)=(k-2) h\left(T_{k}\right)-k+5$ for $h\left(T_{k}\right) \geq 3$ and $k \geq 3$.

\subsection{Wall Capture Time of a Hypercube}

THEOREM 2.5.1. $W_{c_{t}}\left(Q_{n}\right) \leq 2^{n-1}+n-1$ for $n \geq 1$.

PROOF. To begin it is fairly straightforward to see the following: $W_{c_{t}}\left(Q_{0}\right)=1$ and $W_{c_{t}}\left(Q_{1}\right)=1$. By definition, there are $2^{n}$ vertices that each have degree $n$ in any hypercube. Hypercubes are bipartite so the vertices may be split into two disjoint sets $V_{1}$ and $V_{2}$ such that every edge connects a vertex in $V_{1}$ to one in $V_{2}$. This bi-partition is unique and both vertex sets have $2^{n-1}$ vertices. The cop's strategy is to build a wall on every vertex in one of those disjoint sets thereby capturing the robber. Without loss of generality, the cop attempts to build a wall on all the vertices of $V_{1}$.

The only way the robber could hinder this strategy to live longer is by staying on a vertex that the cop wants to build a wall on. Then the robber can only be on one of the vertices the cop does not want him on 
in $V_{1}$. Whichever vertex that is, the cop will start to build walls on all its adjacent vertices but only after all the other vertices in $V_{1}$ have a wall built on them. The robber will skip his turn for the remainder of the game once he is on a vertex in $V_{1}$ and all the other vertices in $V_{1}$ have a wall built on them as otherwise he will lose in one turn if he moves. Then for $Q_{n}$, we have $2^{n-1}-1$ moves to build all the walls in $V_{1}$ except for the one the robber occupies. Then it takes $n$ moves to build walls on all the adjacent vertices of that vertex. That makes a total of $2^{n-1}+n-1$ moves to capture the robber in $Q_{n}$ for $n \geq 1$. Therefore, we have that $W_{c_{t}}\left(Q_{n}\right) \leq 2^{n-1}+n-1$ for $n \geq 1$. 



\section{CHAPTER 3}

\section{Grids}

In this chapter, we will continue our theme of determining the wall capture time of certain graph classes. We will study the wall capture time of infinite grids and tilings of the plane in two dimensions. In Section 3.1, we study the wall capture time of infinite hexagonal grids. In Section 3.2, we study the wall capture time of infinite Cartesian grids. In Section 3.3, we study the wall capture time of infinite triangular grids. In Section 3.4, we study the wall capture time of infinite strong grids. Lastly, in Section 3.5, we study the wall capture time of $n$-layered infinite Cartesian grids.

\subsection{Wall Capture Time of an Infinite Hexagonal Grid}

The infinite hexagonal grid, written $H_{\infty}$, is a tiling of the plane by hexagons with vertices represented as vertices of the hexagons. A drawing of part of the infinite hexagonal grid as seen in Figure 3.1 will be used. The labelling of the coordinates of the grid will follow the rule that the distance between any two adjacent vertices on the $x$-axis or $y$-axis is one unit. 


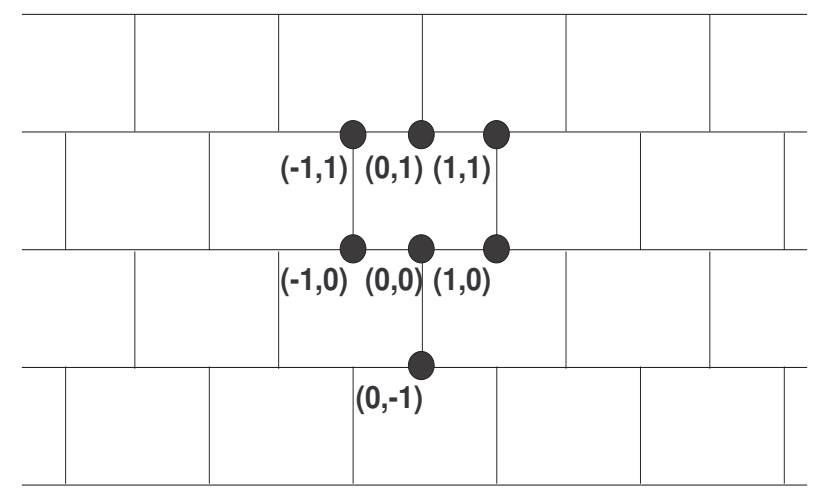

FigurE 3.1. An infinite hexagonal grid with labelled coordinates.

\section{TheOREM 3.1.1. $W_{c_{t}}\left(H_{\infty}\right)=8$.}

PROOF. We begin by showing a strategy for the cop to capture the robber in 8 moves, thus, proving an upper bound of 8 for the wall capture time of $H_{\infty}$. We will present the moves of the cop and robber in a table first. The first column labelled $C$ represents the cop's move. The second column, labelled $R$, represents the robber's move. We will then present the moves again in a numbered format with explanations where we let C: represent the cop's move and R: represent the robber's move. First, in Case A, we will describe how the cop wins if the robber skips his $2^{\text {nd }}$ turn, then in Case B, we will describe how the cop wins if the robber moves up or right on his $2^{\text {nd }}$ turn.

Note that if the robber skips his turn after his $2^{\text {nd }}$ turn but before it is his $5^{t h}$ turn, then the cop can capture him in three moves, resulting in less than 8 moves for the cop. Therefore, the robber will not pursue this move. To see this, after the robber skips his turn, the cop would 
ensure capturing him in three moves by building a wall on an open vertex adjacent to the robber that he has never occupied on a previous turn. The robber would be forced to move to the open adjacent vertex or else be captured next turn. There is only one open adjacent vertex as the cop just built a wall adjacent to the robber and due to the cop's strategy, there was already a wall adjacent to the robber prior to the robber skipping his turn. The cop would then build a wall on the open vertex adjacent to the robber that he did not occupy on the previous turn. The robber would either skip his turn or move to the only open adjacent vertex. The cop would capture him by building a wall adjacent to him.

Case A: The robber skipped his $2^{\text {nd }}$ turn. 


\begin{tabular}{|c|c|}
\hline$C$ & $R$ \\
\hline \hline$(x, y)$ & $(0,0)$ \\
\hline$(-1,0)$ & $(0,0)$ \\
\hline$(2,0)$ & $(0,1)$ \\
\hline$(-1,1)$ & $(1,1)$ \\
\hline$(1,2)$ & $(1,1)$ \\
\hline$(2,1)$ & $(0,1)$ \\
\hline$(0,0)$ & $(0,1)$ \\
\hline$(1,1)$ & Captured \\
\hline
\end{tabular}

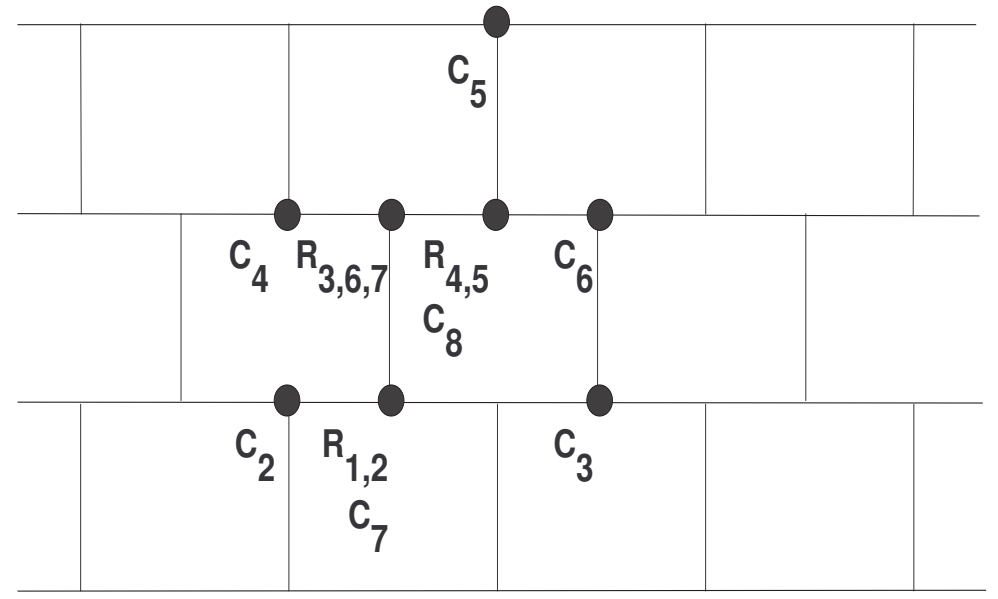

Figure 3.2. Cop and robber's moves in Case A. The labels correspond to the player's turn number and location on the closest vertex up and to the right. When both a cop label and a robber label are needed, the cop label appears below the robber label. The notation $R_{4,5}$ means that the robber's $4^{\text {th }}$ and $5^{\text {th }}$ move are on that vertex. The cop's first move $C_{1}$ is not shown in the figure as it is sufficiently far away from these vertices.

(1) C: This is the cop's first move and because it is an infinite grid, we know it will be wasted. 
$\mathbf{R}$ : The robber plays sufficiently far away from the first cop rendering that first wall useless.

(2) C: Due to symmetry, we can assume that the robber is on a vertex that has two adjacent open vertices, one on its left and one on its right, and one adjacent open vertex above it. The cop then plays on the open vertex to the left of the robber. Here left and right are symmetric so it does not matter which one the cop chooses.

R: The robber skips his turn.

(3) C: The cop plays distance two to the right of the robber.

R: The robber moves up as if he moves right he will be captured in two moves.

(4) C: The cop plays to the left of the robber.

$\mathbf{R}$ : The robber moves to the right.

(5) C: The cop plays directly above the robber.

$\mathbf{R}$ : The robber skips his turn as if he moves to the right, he will be captured in two moves.

(6) C: The cop plays to the right of the robber.

$\mathbf{R}$ : The robber moves left as otherwise, he will be captured next turn.

(7) C: The cop plays directly below the robber.

$\mathbf{R}$ : The robber can only skip his turn. 
(8) C: The cop plays to the right of the robber and the robber is captured. 
Case B: The robber moved up or right on his $2^{\text {nd }}$ turn.

\begin{tabular}{|c|c|}
\hline$C$ & $R$ \\
\hline \hline$(x, y)$ & $(0,0)$ \\
\hline$(-1,0)$ & $(0,1)$ \\
\hline$(1,1)$ & $(-1,1)$ \\
\hline$(-1,2)$ & $(-2,1)$ \\
\hline$(-3,1)$ & $(-2,1)$ \\
\hline$(-2,0)$ & $(-1,1)$ \\
\hline$(0,1)$ & $(-1,1)$ \\
\hline$(-2,1)$ & Captured \\
\hline
\end{tabular}

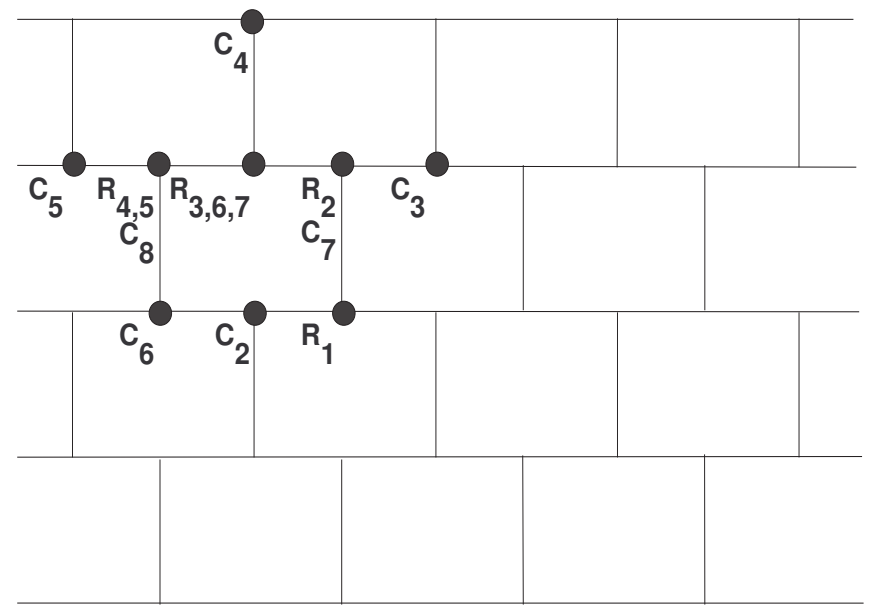

Figure 3.3. Cop and robber's moves in Case B. The labels correspond to the player's turn number and location on the closest vertex up and to the right. When both a cop label and a robber label are needed, the cop label appears below the robber label. The cop's first move $C_{1}$ is not shown in the figure as it is sufficiently far away from these vertices. 
(1) C: This is the cop's first move and because it is an infinite grid, we know it will be wasted.

$\mathbf{R}$ : The robber plays sufficiently far away from the first cop rendering that first wall useless.

(2) C: Due to symmetry, we can assume that the robber is on a vertex that has two adjacent open vertices, one on its left and one on its right, and one adjacent open vertex above it. The cop then plays on the open vertex to the left of the robber. Here left and right are symmetric so it does not matter which one the cop chooses.

$\mathbf{R}$ : The robber can move up or right and as these are symmetric, the robber moves up.

(3) C: The cop plays to the right of the robber.

R: The robber moves left.

(4) C: The cop plays directly above the robber.

R: The robber moves left.

(5) C: The cop plays to the left of the robber.

R: The robber skips his turn as if he moves down, he will be captured in two moves.

(6) C: The cop plays directly below the robber.

$\mathbf{R}$ : The robber moves right as otherwise he will be captured next turn.

(7) C: The cop plays to the right of the robber. 
R: The robber can only skip his turn.

(8) C: The cop plays to the left of the robber and the robber is captured.

Since a strategy for the cop has been shown that requires only 8 moves to capture the smartest robber, we have shown an upper bound of 8 for the $W_{c_{t}}$ of an infinite hexagonal grid. Now we have to show that 8 is also a lower bound for the $W_{c_{t}}$ to show equality by proving the robber cannot be captured in 7 moves.

First notice that since this grid is infinite, the first move for the cop will always be wasted. Now since the degree of each vertex is three, it is clear that four moves are required even if the robber does nothing. If the robber skips his $2^{\text {nd }}$ move, then the robber is guaranteed to have at least one vertex free to move to that has three open vertices in its neighbourhood. This is due to the fact that the only vertex that is shared in the neighbourhoods of the vertices adjacent to the initial vertex the robber occupies is the initial vertex itself. Once the robber moves to this vertex, either none of its neighbours are adjacent to any walls or one of its neighbours is adjacent to one wall or one of its neighbours is adjacent to two walls. We assume the robber moves to this vertex and then we will call these cases 1 through 3, respectively. Note that the cop and robber have each made three moves and it is now the cop's turn. 
Case 1: The robber is on a vertex where none of its neighbours are adjacent to any walls.

We know that the cop did not build any walls within distance one of the initial vertex of the robber due to the case we are in. The robber can get at least five more moves out of the cop by what the robber did at the beginning of the game. The robber will skip his turn once and then move to the adjacent vertex that has no walls built on any of its neighbours, which is a guarantee to exist and just skip his turn for the remainder of the game. This results in 8 moves for the cop.

Case 2: The robber is on a vertex where one of its neighbours is adjacent to one wall.

Due to the case we are in, we know that the cop built one wall within distance one of the initial vertex of the robber and one wall distance two or greater from the initial vertex of the robber. There has to be an adjacent vertex that has no walls built on any of its neighbours so the robber moves there. The cop makes his $5^{\text {th }}$ move. Now, there must exist an adjacent vertex that is not the one the robber just left that also has at least two neighbours with no walls built on them. The robber skips his turn. The vertex the robber is on is adjacent to two vertices that have at least two open neighbours each. The cop's $6^{\text {th }}$ move can only close down one of the options leaving the robber to move to the other and just skip 
his turn for the remainder of the game. This results in 8 moves for the cop.

Case 3: The robber is on a vertex where one of its neighbours is adjacent to two walls.

Due to the case we are in, we know that the cop built two walls within distance one of the initial vertex of the robber. There has to be an adjacent vertex that has no wall built on any of its neighbours so the robber moves there. Again, there must be an adjacent vertex that has no wall built on any of its neighbours so the robber moves there and then just skips his turn for the remainder of the game resulting in 8 moves for the cop.

We have shown that no matter what the cop does, there is always a strategy for the robber to guarantee 8 moves for the cop or that the cop cannot capture him in 7 moves or less. We have also shown that the cop can capture the robber in 8 moves regardless of the strategy the robber chooses. Therefore, we have shown that $W_{c_{t}}\left(H_{\infty}\right)=8$.

\subsection{Wall Capture Time of an Infinite Cartesian Grid}

An infinite Cartesian grid, written $P_{\infty} \square P_{\infty}$, is the Cartesian product of two infinite, two-way paths. We will be labelling the vertices using Cartesian coordinates.

A trap is two walls made by cops on a Cartesian grid such that they share the same $x$ or $y$ coordinate but not both, and are distance two 
apart. The vertex in between these two walls will be called the middle vertex. It is called a trap since if the robber moves onto the middle vertex, then the cop will close the trap by moving to the open vertex that is adjacent to the middle vertex that the robber did not just come from in the last move. The robber cannot move back to his previous vertex by the rules of the game, and then the cop will capture him by playing adjacent to him. Thus, moving into a trap guarantees that the robber will be captured in exactly two turns.
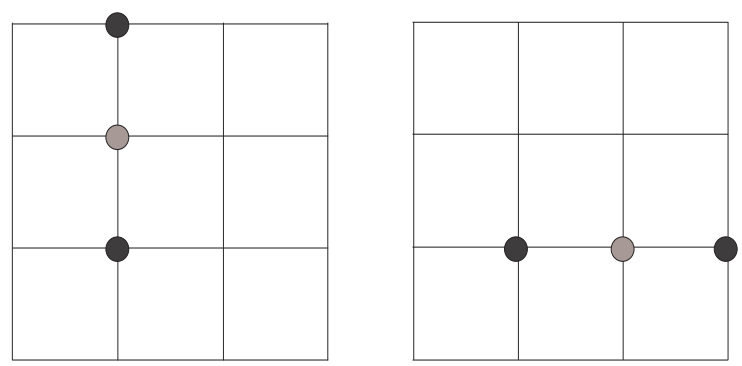

Figure 3.4. The two possible traps the cop can build. The two walls of the trap are in black and the middle vertex is in grey.

TheOREM 3.2.1. $W_{c_{t}}\left(P_{\infty} \square P_{\infty}\right) \leq 14$

Proof. The cop's plan is to confine the robber to a subgraph where he will capture him. The first move by the cop will be wasted as the grid is infinite and so the robber will play so far away from that first wall that is built that it will be of no consequence in capturing the robber. The robber now places himself on a vertex which we will denote as the origin $(0,0)$. The second move for the cop is to play adjacent to the robber and, due to symmetry, it does not matter which vertex the cop chooses so the 
cop will play to the right of the robber on $(1,0)$. Now the robber has three choices since moving up and down are symmetric so we will divide this into cases with Case 1 being that the robber moves up to $(0,1)$, Case 2 being that the robber moves left to $(-1,0)$, and Case 3 being that the robber skips his turn.

Case 1: The robber moved up to $(0,1)$.

The cop plays on $(1,2)$. Now the robber has four choices, but the option of moving right to $(1,1)$ is moving into a trap which results in him being captured in two moves. Therefore, there are only three feasible options and we will once again divide this into cases with Case 1.1 being that the robber moves up to $(0,2)$, Case 1.2 being that the robber moves left to $(-1,1)$, and Case 1.3 being that the robber skips his turn.

Case 1.1: The robber moved up to $(0,2)$.

The cop plays on $(-1,3)$. Now the robber has three options. We will again divide this into three cases with Case 1.1.1 being that the robber moves up to $(0,3)$, Case 1.1 .2 being that the robber moves left to $(-1,2)$, and Case 1.1.3 being that the robber skipped his turn.

Case 1.1.1: The robber moved up to $(0,3)$.

The robber will be captured in 12 moves or less in this case. We will now start the count again for the moves of the cop and robber.

(1) C: The cop plays on $(0,4)$. 
$\mathbf{R}$ : The robber will not skip his turn since then the cop will play on $(1,3)$ and force him to move back to $(0,2)$. This would be the same situation as Case 1.1.3, except one more move would have been played and the option of moving back up to $(0,3)$ is gone as the robber would be captured in two moves following that. This will be shown in Case 1.1.3 to still result in 11 moves or less, thus, he moves right to $(1,3)$.

(2) C: The cop plays on $(2,4)$.

$\mathbf{R}$ : The robber will not skip his turn as the cop would then play on $(2,3)$. This would allow the robber to move back to $(0,3)$ which is bad since the cop would play on $(0,2)$ forcing the robber to skip his next turn and then the cop would play on $(1,3)$, thereby, capturing him. The robber could also move up into the trap but would then be captured in two turns. Lastly, the robber could also skip his turn again which would allow the cop to force him to backtrack to $(0,3)$ or move into the trap, both of which have been shown to be bad. So, if the robber skips his turn he gets captured in at most 10 moves. The robber will not move up into the trap as then he gets captured in 8 moves. Thus, the robber moves right to $(2,3)$.

(3) C: The cop plays on $(3,3)$. 
$\mathbf{R}$ : The robber will not skip his turn as then the cop would play on $(2,2)$ which would force the robber to backtrack to $(1,3)$ and be captured in at most 11 moves. Therefore, the robber moves down to $(2,2)$.

(4) C: The cop plays on $(3,1)$.

$\mathbf{R}$ : The robber will not move right into the trap as this results in 10 moves. If he skips his turn or moves down, then he will be captured in 12 moves. If he skips his turn, then the cop will play on $(2,1)$ and then he will skip his turn again followed by moving to the open adjacent vertex and be captured in 12 moves. So we will say the robber moves down to $(2,1)$.

(5) $\mathbf{C}$ : The cop plays on $(2,0)$.

$\mathbf{R}$ : The robber will not move left to $(1,1)$ as then the cop will play on $(0,1)$ forcing the robber to skip his turn at which point the cop will play on $(2,1)$ and capture him in 11 moves. Therefore, the robber will skip his turn since then the cop will play on $(2,2)$ forcing the robber to move left as above resulting in the robber being captured in 12 moves.

(6) C: The cop plays on $(2,2)$.

$\mathbf{R}$ : The robber has to move left to $(1,1)$ or be captured next turn.

(7) C: The cop plays on $(0,1)$. 
$\mathbf{R}$ : The robber can only skip his turn.

(8) C: The cop plays on $(2,1)$ and the robber is captured.

The robber is captured in 12 moves; thus, this is not the optimal strategy for the robber and this concludes Case 1.1.1.

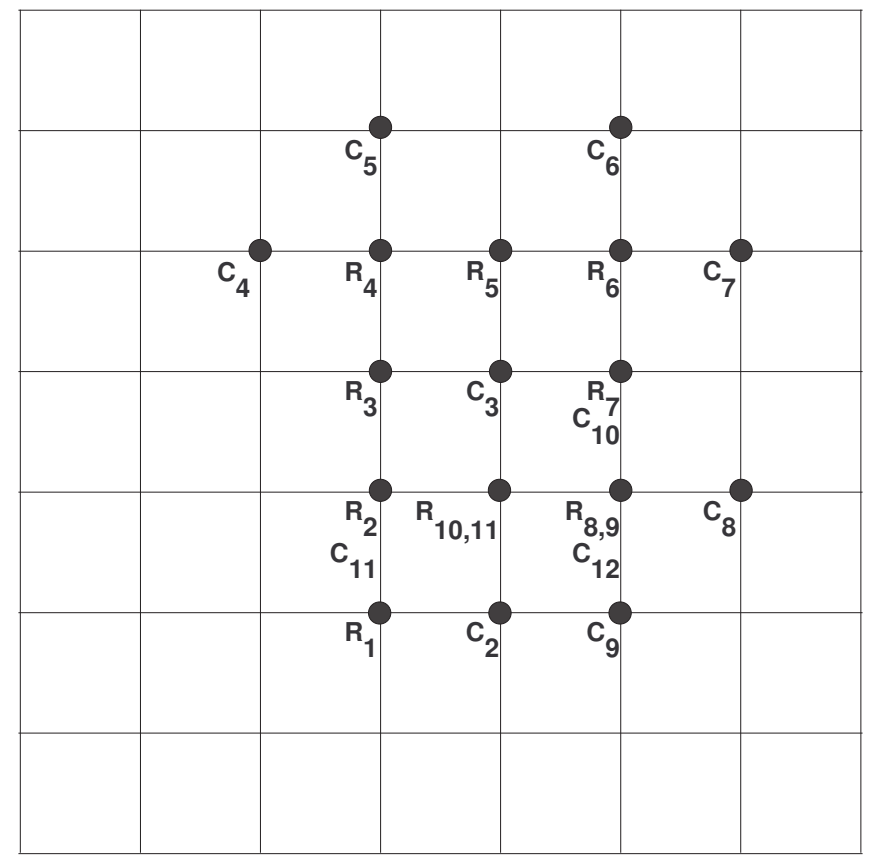

Figure 3.5. Cop and robber's moves in Case 1.1.1. The labels correspond to the player's turn number and location on the closest vertex up and to the right. When both a cop label and a robber label are needed, the cop label appears below the robber label. $R_{8,9}$ means that the robber's $8^{\text {th }}$ and $9^{\text {th }}$ moves are on that vertex. The cop's first move $C_{1}$ is not shown in the figure as it is sufficiently far away from these vertices.

Case 1.1.2: The robber moved left to $(-1,2)$.

The robber will be captured in 13 moves or less in this case. We will now start the count again for the moves of the cop and robber.

(1) C: The cop plays on $(-2,2)$. 
$\mathbf{R}$ : The robber will not skip his turn as then the cop would play on $(-1,1)$ forcing the robber to move to $(0,2)$. The cop would then play on $(0,3)$ forcing the robber to move down to $(0,1)$ or be captured in two turns. The cop would then play on $(0,0)$ after which the robber would skip his turn or he would be captured in two moves. The cop would then play on $(0,2)$ forcing the robber to move into the trap at $(1,1)$ and be captured in a total of 11 moves. Thus, the robber moves to $(-1,1)$.

(2) C: The cop plays on $(-2,0)$.

$\mathbf{R}$ : The robber will not move left into the trap. If the robber moves right to $(0,1)$ then the cop would play on $(0,0)$ and from there, capture him in at most 11 moves total. If the robber skips his turn then the cop would play on $(-1,0)$ and from there, capture him in at most 12 moves. Thus, the robber moves down to $(-1,0)$.

(3) C: The cop plays on $(-1,-1)$.

$\mathbf{R}$ : The robber will not skip his turn as then the cop would play on $(0,0)$ and capture him in at most 12 moves. Therefore, the robber moves right to $(0,0)$.

(4) C: The cop plays on $(0,-1)$.

$\mathbf{R}$ : The robber will not skip his turn as then the cop would play on $(0,1)$ and capture him in at most 11 moves. Thus, the robber moves up to $(0,1)$. 
(5) C: The cop plays on $(-1,1)$.

$\mathbf{R}$ : The robber will not move right to $(1,1)$ as it is a trap. The robber will not move up to $(0,2)$ as then the cop would play on $(0,3)$ and from there capture him in at most 12 moves. Thus, the robber skips his turn.

(6) C: The cop plays on $(0,2)$.

$\mathbf{R}$ : The robber skips his turn as otherwise, he will be captured in two moves.

(7) C: The cop plays on $(1,1)$.

R: The robber moves down to $(0,0)$ as, otherwise, he will be captured next turn.

(8) C: The cop plays on $(-1,0)$.

$\mathbf{R}$ : The robber must skip his turn.

(9) C: The cop plays on $(0,1)$ and the robber is captured.

The robber is captured in 13 moves; thus, this is not one of the robber's optimal strategies and this concludes Case 1.1.2. 


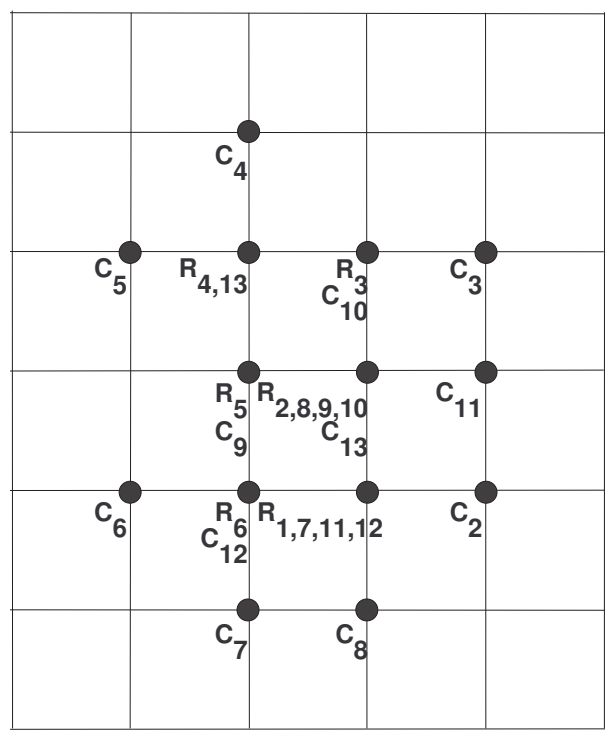

Figure 3.6. Cop and robber's moves in Case 1.1.2.

Case 1.1.3: The robber skipped his turn.

The cop plays on $(-1,1)$. The robber would then have four choices.

(1) If the robber moved down to $(0,1)$ then the cop would play on $(0,0)$ and from there capture the robber in at most 9 moves.

(2) If the robber moved up to $(0,3)$ then it would be the same scenario as in Case 1.1.1 which would result in 13 moves as a result of the extra move taken by the cop when the robber skipped his turn.

(3) If the robber moved left to $(-1,2)$ then he would be moving into a trap and be captured in 7 moves total.

(4) If the robber skipped his turn then the cop would play on $(0,3)$ and from there capture the robber in at most 10 moves.

The robber is captured in 13 or less moves; thus, this is not one of the optimal strategies for the robber and this concludes Case 1.1.3. 
Case 1.1.1, Case 1.1.2, and Case 1.1.3 have been resolved and thus, this concludes Case 1.1.

Case 1.2: The robber moved left to $(-1,1)$.

The cop plays on $(-2,1)$. Now, the robber has two options as up and down are symmetric. We will divide this into two cases, with Case 1.2.1 being that the robber moves up to $(-1,2)$ and Case 1.2 .2 being that the robber skips his turn.

Case 1.2.1: The robber moved up to $(-1,2)$.

The robber will be captured in 13 moves or less in this case. We will now start the count again for the moves of the cop and robber.

(1) $\mathbf{C}$ : The cop plays on $(-2,3)$.

$\mathbf{R}$ : The robber will not move left into the trap. The robber will not move right to $(0,2)$ as then the cop would play on $(0,3)$. From there, the robber would move down to $(0,1)$ and the cop would play on $(0,0)$, forcing the robber to move to $(-1,1)$. The cop would play on $(-1,0)$ and from there capture the robber in at most 13 moves. The robber will not skip his turn as then the cop would play on $(0,2)$ and from there capture the robber in at most 12 moves. Thus, the robber moves up to $(-1,3)$.

(2) C: The cop plays on $(-1,4)$. 
$\mathbf{R}$ : The robber will not skip his turn as then the cop would play on $(-1,2)$ and from there capture the robber in at most 12 moves. Thus, the robber moves right to $(0,3)$.

(3) C: The cop plays on $(1,4)$.

$\mathbf{R}$ : The robber will not move up or right into the traps. The robber will not skip his turn as then the cop would play on $(-1,2)$ and from there capture the robber in at most 12 moves. Thus, the robber moves down to $(0,2)$.

(4) $\mathbf{C}$ : The cop plays on $(0,1)$.

R: The robber can now either move left or he can skip his turn. Both of these options result in 13 moves for the cop so without loss of generality, the robber moves left to $(-1,2)$.

(5) C: The cop plays on $(0,2)$.

$\mathbf{R}$ : The robber will skip his turn as, otherwise, he will be captured in two moves.

(6) $\mathbf{C}$ : The cop plays on $(-1,3)$.

$\mathbf{R}$ : The robber skips his turn again for the same reasons.

(7) C: The cop plays on $(-1,1)$.

$\mathbf{R}$ : The robber moves left into the trap at $(-2,2)$ as otherwise, he will be captured next turn.

(8) C: The cop plays on $(-3,2)$.

$\mathbf{R}$ : The robber must skip his turn. 
(9) C: The cop plays on $(-1,2)$ and the robber is captured.

The robber is captured in 13 moves; thus, this is not one of the robber's optimal strategies and this concludes Case 1.2.1.

Case 1.2.2: The robber skipped his turn.

The robber will be captured in 13 moves or less in this case. We will now start the count again for the moves of the cop and robber.

(1) C: The cop plays on $(-1,2)$.

$\mathbf{R}$ : The robber will not move right to $(0,1)$ as then the cop would play on $(0,0)$ forcing the robber to skip his next turn or be captured in two moves. The cop would then play on $(-1,1)$ and from there capture the robber in at most 10 moves. The robber will not skip his turn as then the cop would play on $(-1,0)$, forcing the robber to move right to $(0,1)$ and from there like above, he would capture the robber in at most 10 moves. Thus, the robber moves down to $(-1,0)$.

(2) C: The cop plays on $(-2,-1)$.

$\mathbf{R}$ : The robber will not move left into the trap. The robber will not move right as then the cop would play on $(0,-1)$ and from there capture the robber in at most 12 moves. The robber could skip his turn as then the cop would play on $(-1,-1)$ and from there capture the robber in at most 13 moves. But, since the robber 
can get 13 moves from moving down too, the robber moves down to $(-1,-1)$.

(3) C: The cop plays on $(-1,-2)$.

$\mathbf{R}$ : The robber will not skip his turn as then the cop would play on $(0,-1)$ and capture the robber in at most 12 moves. Thus, the robber moves right to $(0,-1)$.

(4) C: The cop plays on $(1,-2)$.

$\mathbf{R}$ : The robber will not move right or down into the traps. The robber could skip his turn as then the cop would play on $(0,0)$ and from there capture the robber in at most 13 moves. But, since moving up results in 13 moves too, the robber moves up to $(0,0)$.

(5) $\mathbf{C}$ : The cop plays on $(0,1)$.

$\mathbf{R}$ : The robber can either skip his turn or move left as both result in 13 moves for the cop. Thus, the robber moves left to $(-1,0)$.

(6) C: The cop plays on $(-1,-1)$.

$\mathbf{R}$ : The robber skips his turn as otherwise he will be captured in two moves.

(7) C: The cop plays on $(0,0)$.

$\mathbf{R}$ : The robber skips his turn again for the same reasons as above.

(8) C: The cop plays on $(-2,0)$. 
$\mathbf{R}$ : The robber skips his turn as, no matter what, he will be captured next turn.

(9) C: The cop plays on $(-1,1)$ and the robber is captured.

The robber is captured in 13 moves; thus, this is not one of the robber's optimal strategies and this concludes Case 1.2.2.

Case 1.2.1, and Case 1.2.2 have been resolved and thus, this concludes Case 1.2.

Case 1.3: The robber skipped his turn.

The cop plays on $(-1,1)$. Now the robber has two options as up and down are symmetric. We will divide this into two cases with Case 1.3.1 being that the robber moves up to $(0,2)$ and Case 1.3 .2 being that the robber skips his turn.

Case 1.3.1: The robber moved up to $(0,2)$.

The robber will be captured in 14 moves or less in this case. We will now start the count again for the moves of the cop and robber.

(1) C: The cop plays on $(0,3)$.

$\mathbf{R}$ : The robber will not skip his turn as then the cop would play on $(-1,2)$ and from there capture the robber in at most 9 moves. Thus, the robber moves left to $(-1,2)$.

(2) C: The cop plays on $(-2,3)$.

$\mathbf{R}$ : The robber will not move up into the trap. The robber will not skip his turn as then the cop would play on $(-2,2)$ and from 
there capture the robber in at most 10 moves. Thus, the robber moves left to $(-2,2)$.

(3) C: The cop plays on $(-3,2)$.

$\mathbf{R}$ : The robber will not skip his turn for the same reason he did not skip his fifth turn. Thus, the robber moves down to $(-2,1)$.

(4) C: The cop plays on $(-3,0)$.

$\mathbf{R}$ : The robber will not skip his turn for the same reason he did not skip his sixth turn. Thus, the robber moves down to $(-2,0)$.

(5) C: The cop plays on $(-2,-1)$.

$\mathbf{R}$ : The robber will not skip his turn for the same reason he did not skip his seventh turn as the cop would capture him in at most 13 moves. Thus, the robber moves right to $(-1,0)$.

(6) C: The cop plays on $(0,-1)$.

R: The robber skips his turn as otherwise he will be captured in two moves.

(7) C: The cop plays on $(0,0)$.

$\mathbf{R}$ : The robber skips his turn again for the same reasons as above.

(8) C: The cop plays on $(-2,0)$.

$\mathbf{R}$ : The robber moves down to $(-1,-1)$ as otherwise, he will be captured next turn.

(9) C: The cop plays on $(-1,-2)$. 
$\mathbf{R}$ : The robber must skip his turn.

(10) C: The cop plays on $(-1,0)$ and captures the robber.

The robber is captured in 14 moves and this concludes Case 1.3.1.

Case 1.3.2: The robber skipped his turn.

The robber will be captured in 13 moves or less in this case. We will now start the count again for the moves of the cop and robber.

(1) C: The cop plays on $(0,0)$.

$\mathbf{R}$ : The robber will not move right into the trap. The robber will not skip his turn as then the cop would play on $(0,2)$ and from there capture the robber in at most 8 moves. Thus, the robber moves up to $(0,2)$.

(2) C: The cop plays on $(0,3)$.

$\mathbf{R}$ : The robber will not skip his turn as then the cop would play on $(-1,2)$ and from there capture the robber in at most 9 moves. Thus, the robber moves left to $(-1,2)$.

(3) C: The cop plays on $(-2,3)$.

$\mathbf{R}$ : The robber will not move up into the trap. The robber will not skip his turn as then the cop would play on $(-2,2)$ and from there capture the robber in at most 11 moves. Thus, the robber moves left to $(-2,2)$.

(4) C: The cop plays on $(-3,2)$. 
$\mathbf{R}$ : The robber will not skip his turn for the same reason he did not skip his sixth turn. Thus, the robber moves down to $(-2,1)$.

(5) C: The cop plays on $(-3,0)$.

$\mathbf{R}$ : The robber will not skip his turn for the same reason he did not skip his seventh turn. Thus, the robber moves down to $(-2,0)$.

(6) C: The cop plays on $(-2,-1)$.

$\mathbf{R}$ : The robber will not move right to $(-1,0)$ as then the cop would play on $(-1,-1)$ forcing the robber to skip his next turn and then capturing him by playing on $(-2,0)$. Thus, the robber skips his turn.

(7) C: The cop plays on $(-2,1)$.

R: The robber has to move right to $(-1,0)$ or he will be captured next turn.

(8) C: The cop plays on $(-1,-1)$.

$\mathbf{R}$ : The robber must skip his turn.

(9) C: The cop plays on $(-2,0)$ and captures the robber.

The robber is captured in 13 moves; thus, this is not one of the robber's optimal strategies and this concludes Case 1.3.2.

Case 1.3.1, and Case 1.3.2 have been resolved and thus, this concludes Case 1.3. 
Case 1.1, Case 1.2, and Case 1.3 have been resolved and thus, this concludes Case 1.

Case 2: The robber moved left to $(-1,0)$.

The robber will be captured in 14 moves or less in this case. We will now start the count again for the moves of the cop and robber.

(1) C: The cop plays on $(-2,0)$.

$\mathbf{R}$ : The robber has two options. He can move up or down as they are symmetric and he can skip his turn. He will not move up or down since then the cop would play the same strategy he plays when the robber skips his turn except he will not have built a wall on $(-1,-1)$. Thus, the robber skips his turn.

(2) C: The cop plays on $(-1,-1)$.

$\mathbf{R}$ : The robber will not skip his turn since then the cop would play on $(0,1)$ forcing the robber to move up to $(-1,1)$ and capture him in at most 12 moves. The robber will not move back right to $(0,0)$ since then the cop would play on $(0,1)$ and capture him in at most 13 moves. Thus, the robber moves up to $(-1,1)$.

(3) C: The cop plays on $(-2,2)$.

$\mathbf{R}$ : The robber will not move left into the trap. The robber will not skip his turn as then he would be in the same situation as if he skipped his last turn. The robber will not move right since 
then the cop would play on $(1,2)$ and capture him in at most 13 moves. Thus, the robber moves up to $(-1,2)$.

(4) C: The cop plays on $(-1,3)$.

R: The robber will not skip his turn since then the cop would play on $(0,2)$ forcing the robber to move back to $(-1,1)$ and he would be surrounded by traps and be captured in at most 12 moves. Thus, the robber moves right to $(0,2)$.

(5) C: The cop plays on $(1,3)$.

$\mathbf{R}$ : The robber will not move up into the trap. The robber will not skip his turn since then the cop would play on $(0,1)$ and capture the robber in at most 12 moves. Thus, the robber moves right to $(1,2)$.

(6) C: The cop plays on $(2,2)$.

$\mathbf{R}$ : The robber can skip his turn or move down as both result in 14 moves. Thus, without loss of generality, the robber moves down to $(1,1)$.

(7) C: The cop plays on $(2,1)$.

$\mathbf{R}$ : The robber will not skip his turn since then the cop would play on $(0,1)$ and capture the robber in at most 12 moves. Thus, the robber moves left to $(0,1)$.

(8) C: The cop plays on $(-1,1)$. 
$\mathbf{R}$ : The robber will not move down since then the cop would play on $(0,-1)$ and capture him in at most 13 moves. The robber can move up or skip his turn as both result in 14 moves. Thus, the robber moves up to $(0,2)$.

(9) C: The cop plays on $(1,2)$.

$\mathbf{R}$ : The robber will skip his turn since otherwise he will be captured in two moves.

(10) C: The cop plays on $(0,1)$.

$\mathbf{R}$ : The robber can either move up to $(0,3)$ or skip his turn since both result in 14 moves. Thus, the robber moves up to $(0,3)$.

(11) C: The cop plays on $(0,4)$.

R: The robber must skip his turn.

(12) $\mathbf{C}$ : The cop plays on $(0,2)$ and the robber is captured.

The robber is captured in 14 moves and this concludes Case 2. 


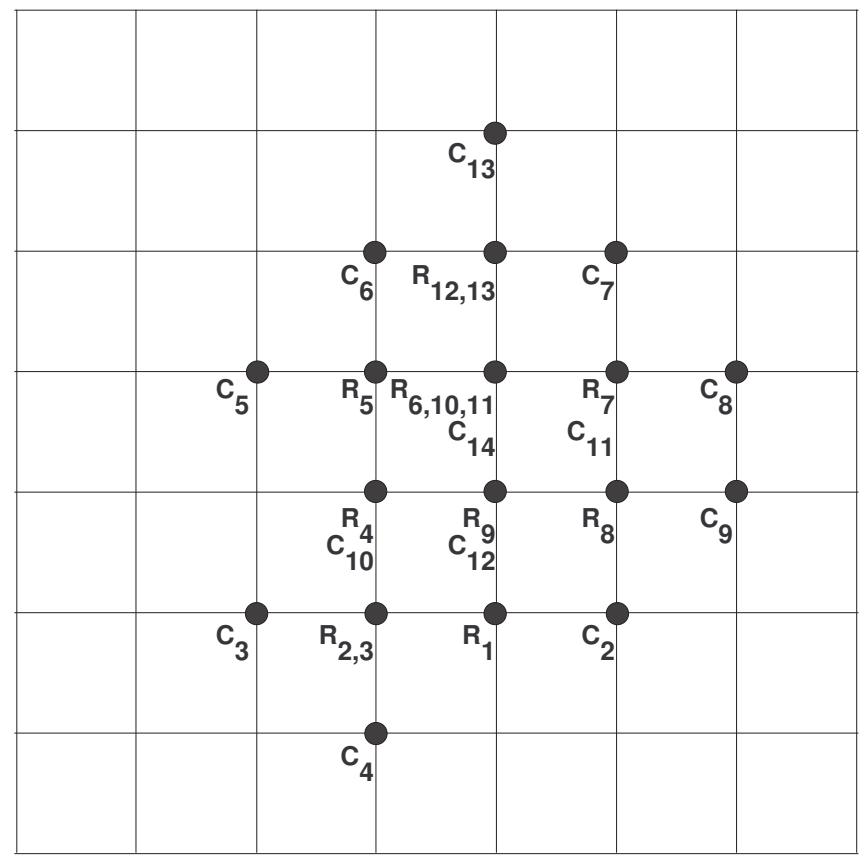

Figure 3.7. Cop and robber's moves in Case 2.

Case 3: The robber skipped his second turn.

The robber will be captured in 14 moves or less in this case. We will now start the count again for the moves of the cop and robber.

(1) C: The cop plays on $(-1,0)$.

$\mathbf{R}$ : If the robber moves up then the cop plays above him. If the robber moves down then the cop plays below him. Both these situations are symmetric to the situation in Case 1.3 which results in 14 total moves for the cop. The robber skipping his turn also results in 14 moves so the robber skips his turn.

(2) C: The cop plays on $(0,-1)$. 
$\mathbf{R}$ : The robber will not skip his turn as then the cop would capture him next turn. Thus, the robber moves up to $(0,1)$.

(3) C: The cop plays on $(1,2)$.

$\mathbf{R}$ : The robber will not move right into the trap. The robber will not skip his turn as then the cop would play on $(-1,2)$, surrounding the robber with traps and from there capture the robber in at most 10 moves. The robber can either move up or left then. These are symmetric as the cop will not let the wall he built on $(0,-1)$ come into play again. Thus, the robber moves up to $(0,2)$.

(4) C: The cop plays on $(0,3)$.

R: The robber will not skip his turn as then the cop would play on $(-1,2)$ and force the robber to move back to $(0,1)$ and be surrounded by traps. Thus, the robber moves left to $(-1,2)$.

(5) C: The cop plays on $(-2,3)$.

$\mathbf{R}$ : The robber will not move up into the trap. The robber will not skip his turn as then the cop would play on $(-2,1)$ and capture the robber in at most 13 moves. Thus, the robber left to $(-2,2)$.

(6) C: The cop plays on $(-3,2)$.

$\mathbf{R}$ : The robber will not skip his turn as then the cop would play on $(-2,1)$ and capture the robber in at most 13 moves. Thus, the robber moves down to $(-2,1)$.

(7) C: The cop plays on $(-3,0)$. 
$\mathbf{R}$ : The robber will not move left or down into the traps. The robber can move right or skip his turn as both result in 14 moves. If the robber moves right then the cop would play on $(0,1)$ forcing the robber to move up or skip his turn both again which result in 14 moves. Let us say then that the robber skipped his turn initially.

(8) C: The cop plays on $(-1,2)$.

$\mathbf{R}$ : The robber skips his turn as he is surrounded by traps.

(9) C: The cop plays on $(-1,1)$.

$\mathbf{R}$ : The robber skips his turn again as he is surrounded by traps.

(10) C: The cop plays on $(-2,0)$.

$\mathbf{R}$ : The robber can either skip his turn or move left as they both result in 14 moves so let the robber move left to $(-3,1)$.

(11) C: The cop plays on $(-4,1)$.

$\mathbf{R}$ : The robber must skip his turn.

(12) C: The cop plays on $(-2,1)$ and captures the robber.

The robber is captured in 14 moves and this concludes Case 3.

All the cases have been shown to result in 14 moves or less for the cop to win. Therefore, $W_{c_{t}}\left(P_{\infty} \square P_{\infty}\right) \leq 14$. 


\subsection{Wall Capture Time of an Infinite Triangular Grid}

The infinite triangular grid, written $\triangle_{\infty}$, is a tiling of the plane by triangles with vertices represented as vertices of the triangles. A drawing of part of the infinite triangular grid as seen in Figure 3.8 will be used.

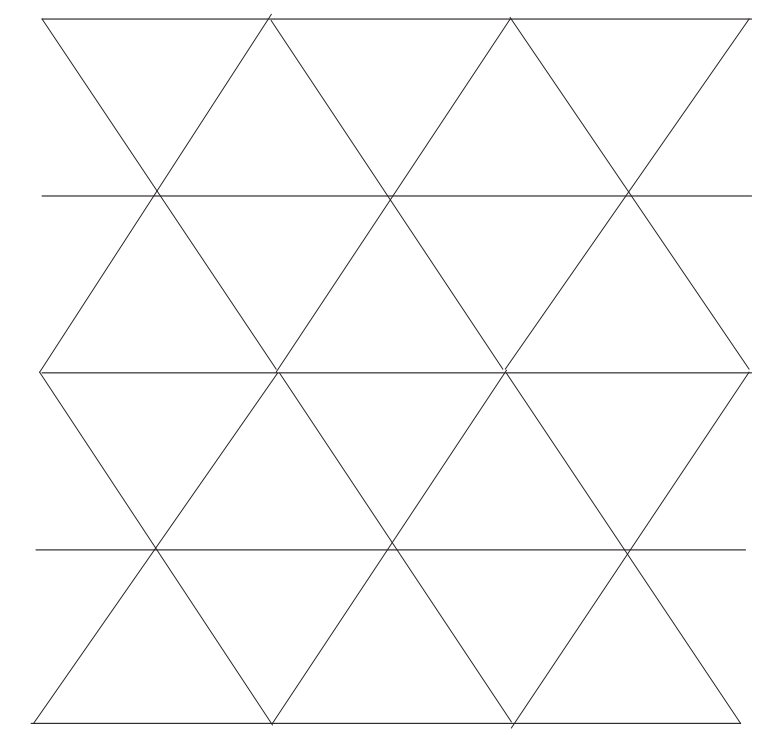

Figure 3.8. An infinite triangular grid. Vertices are omitted.

THEOREM 3.3.1. $W_{c_{t}}\left(\triangle_{\infty}\right) \leq 138$.

Proof. The cop will first trap the robber in a hexagon with lengths of sides $3,21,21,21,21$, and 3 . The cop will build the hexagon, taking the robber's vertex as the centre of this hexagon so that he is distance 11 from each of its walls. The robber will not move backwards or skip his turn while the hexagon is being built as this will allow the cop to build a smaller hexagon and thus, use fewer moves to capture the robber. We will describe the corners of the hexagon as follows: TLC, TRC, LC, RC, 
BLC, BRC with $\mathrm{T}$ standing for top, B standing for bottom, R standing for right, L standing for left, and $\mathrm{C}$ standing for corner. The first 9 moves for the cop are as follows:

(1) The first move of the cop is wasted as we are playing on an infinite grid, so the robber will just play so far away from the first cop that the wall he builds will be useless.

(2) The cop plays one up and right of the LC.

(3) The cop plays one down and right of the LC.

(4) The cop plays one up and left of the RC.

(5) The cop plays one down and left of the RC.

(6) The cop plays on the TLC.

(7) The cop plays on the TRC.

(8) The cop plays on the BLC.

(9) The cop plays on the BRC.

The robber must have moved towards one of the sides of the hexagon in these first 9 moves. If he moved up, then the cop's $10^{t h}$ move is to play on the vertex two down and left of the TRC. If he moved down, then the cop's $10^{t h}$ move is to play on the vertex two up and right of the BLC. If the robber went straight left or right without any diagonal movements then the cop would not have had to play there so the robber would not move like that. Otherwise, the cop could build a smaller hexagon. Then we know that the robber will move either up or down towards the TLC 
or TRC or the BLC or BRC. Now the robber is distance two away from a side (only one) and it is the cop's turn and thus, the cop can stop him getting on the sides of the hexagon.

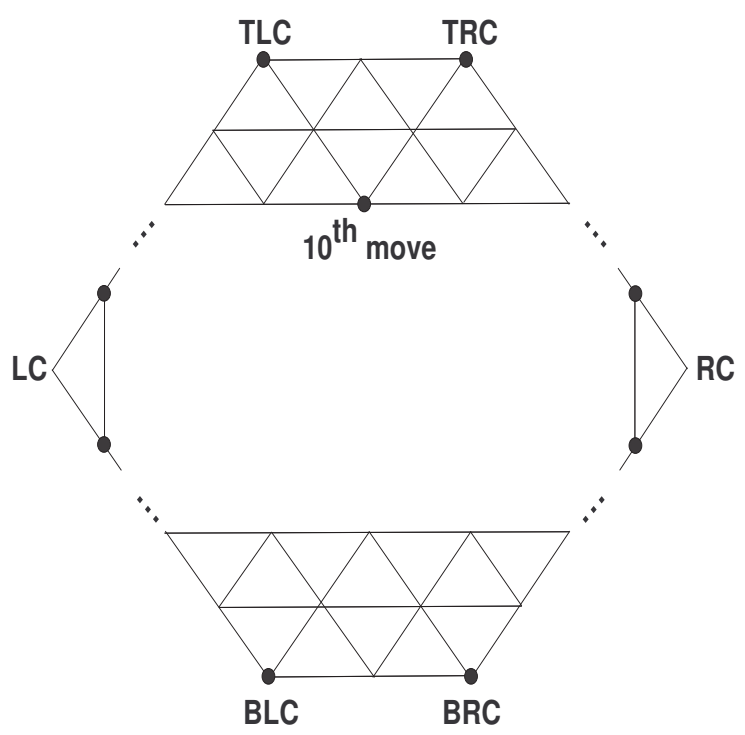

FiguRE 3.9. The first 10 moves of the cop shown as black vertices, with the first move not shown as it is far away from the hexagon. Also, the assumption here for the tenth move is that the robber moved up towards the TLC or TRC.

As the robber runs along the sides of the hexagon, the cop will gain a move on the robber at the $\mathrm{LC}$ and $\mathrm{RC}$ due to the walls that were built at the start. The cop will use these two extra moves to build a wall on the vertex in between the TLC and TRC and a wall on the vertex in between the BLC and BRC in the order that the robber will approach these vertices.

The robber may be able to move toward the centre of the hexagon after most of the sides have been built by the cop in order to gain some extra moves. It is difficult to know exactly when this may happen and 
in which exact direction the robber would move at the start of the game and at this point in the game. Therefore, we will assume that the cop will build the entire hexagon even if the robber moves toward the centre of the hexagon early. Thus, the robber will force the cop to build a wall inside the hexagon on his $10^{\text {th }}$ move as described above. We will assume also that the robber can be anywhere inside this hexagon after it is built. The robber will be in the centre of the hexagon as if he is near any of the sides, it will allow the cop to use those sides to trap him in a smaller subgraph in this next phase.

The cop will then confine the robber to a parallelogram with diagonals of length 23 and the other two sides of length five. We will assume, without loss of generality, that the diagonals go from the top left side of the hexagon to the bottom right side of the hexagon. Since both the top left side and bottom right side of the hexagon are distance 11 from the centre, the diagonal between them is of length 23. The two diagonals of length 23 will be built distance five apart. One will be built distance two from the robber on his left and the other will be built distance three from the robber on his right. This guarantees that the robber is always at least distance two away from either diagonal with it being the cop's turn. The first wall built on these diagonals will be built on the left one since it is only distance two away from the robber. Thus, the robber will never be able to occupy a vertex on either diagonal or leave the 
parallelogram. The two sides of length five are already built as they are part of the sides of the hexagon. Once this parallelogram is completely built, we will assume that the robber is in the best position possible. Therefore, he will be in the centre of this parallelogram.

The cop will then be building two sides parallel to the sides of the hexagon of length three to trap the robber in a $5 \times 4$ parallelogram. The first of these two sides will be built distance one away from the robber, down and to his right, since it is possible to stop him bypassing the side as there are only three open vertices to cover. Thus, the cop will play adjacent to the robber on this side he will be building as seen in Figure 3.10. The robber can move so that the side must actually be built as parallel to the sides of the parallelogram of length five. This does not matter as in either case the cop will trap the robber in a $5 \times 4$ parallelogram. From here, the other side will be built distance two away from the robber's starting position in the parallelogram on the opposite side of the first side built and parallel to the first side built. Now the robber is trapped in a $5 \times 4$ parallelogram. The robber will move back to the vertex he started on after the large parallelogram's sides were built. The cop will capture the robber in at most four moves inside this small parallelogram, no matter where the robber moves inside it as there are only six open vertices and the cop can cut out one of two vertices that are distance three apart. 


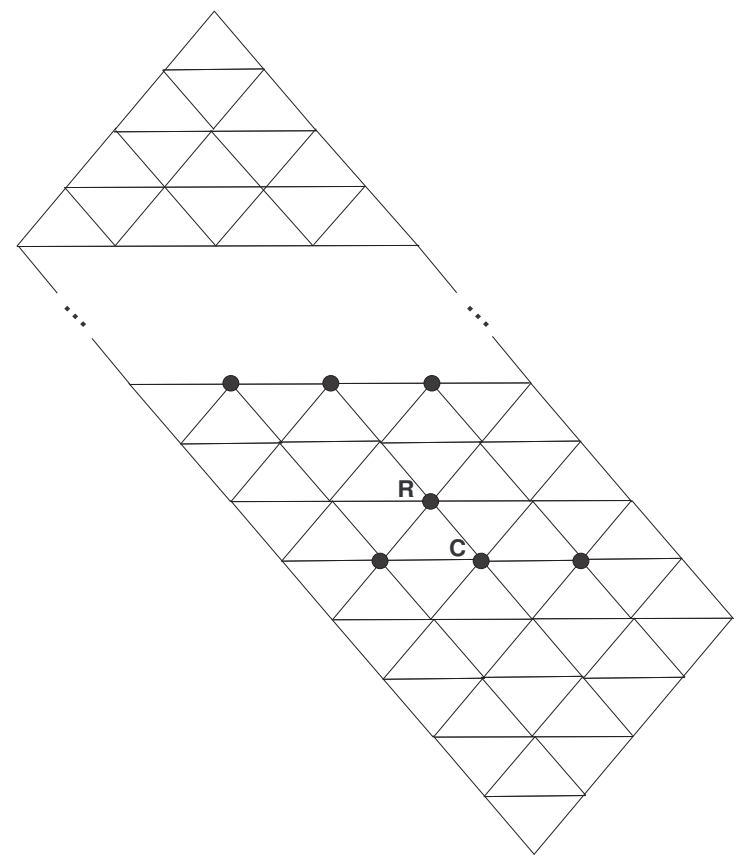

FiguRE 3.10. The parallelogram that is built with the $5 \times 4$ parallelogram built inside it with the robber's starting vertex labelled $\mathrm{R}$ and the cop's first move labelled $\mathrm{C}$ and the rest of his moves to construct the parallelogram not labelled. This figure assumes the robber moves in a way that allows the sides of the parallelogram to be parallel to the sides of the hexagon of length three.

Now we will sum up all the moves of the cop. The cop took one wasted move at the start. He then takes $21 \times 4+3+3+1-6=85$ moves (subtract six for counting six vertices twice) to build the sides of the hexagon and a wall on the vertex on his $10^{t h}$ move. Followed by 42 moves to build the two diagonals of length 23 of the large parallelogram. Six moves to complete the sides of the $5 \times 4$ parallelogram. Then, four moves to capture the robber inside the $5 \times 4$ parallelogram. Thus, it took the cop $1+85+42+6+4=138$ moves to capture the robber. The proof now follows. 


\subsection{Wall Capture Time of an Infinite Strong Grid}

The infinite strong grid, written $P_{\infty} \otimes P_{\infty}$, is the strong product of two infinite, two-way paths. We will be labelling the vertices using Cartesian coordinates. We rely on the following fact from [2]. Note that in the Angel Problem, the Angel (that is robber in our case), unlike in Wall Cops and Robbers, is allowed to move to a vertex he occupied in the previous turn. However, such moves will only lengthen the play of the game. Hence, bounds on the length of the Angel Problem will also be bounds on the wall capture time played on the infinite strong grid.

LEMMA 3.4.1. [2] On the infinite strong grid, the cop can confine the robber to a $35 \times 35$ box by building three walls in each corner on his first 12 moves. The cop can confine the robber from there as he can always stop the robber reaching a side of this box if the robber is distance five from a side and it is the cop's turn.

We now turn to the main result of this section.

Theorem 3.4.2. $W_{c_{t}}\left(P_{\infty} \otimes P_{\infty}\right) \leq 246$.

ProOF. Without loss of generality, we will assume that after the cop plays his first turn, that the robber will place himself sufficiently far away that this first wall will play no part in capturing the robber. Thus, we will count this move only at the end in the total number of moves but will not count it in our ordered turns for the cop below. The cop's plan is 
to capture the robber in a $35 \times 32$ box first and then capture the robber inside that box. The robber's best strategy has to be to move in one direction such as up or right, and or diagonally up and right so as to make it difficult for the cop to trap him. Otherwise, as moving back in the opposite direction while this box is being set up will just waste the robber's turn and possibly allow the cop to build a smaller box to contain the robber. If the robber skips his turn several times, then it will allow the cop to either encroach the sides of the box or if they are already built, then it will allow the cop to encroach the vertical or horizontal walls that enclose the robber after that. Therefore, it is not a good idea for the robber to skip his turn until he knows he will be captured and there are few turns remaining because even doing it once will allow the cop to be one turn ahead of the robber which may also result in fewer moves needed.

The cop will start by building the corners of a $35 \times 35$ box as in Lemma 3.4.1 and once a few moves have been made it will be clear what direction, if any, the robber has chosen to move in which will allow the cop to encroach one of the sides in three steps making it a $35 \times 32$ box (or encroach two of the sides in for a total of three steps, also making it a $35 \times 32$ box). The box must start as a $35 \times 35$ box since the cop requires 12 moves before the robber can be at a distance of five moves from any 
side, thus, making it necessary that the robber start at a distance of 17 moves from any side which a $35 \times 35$ box ensures.

We will assume that the robber is moving diagonally opposite of the corner in which the cop first plays as this makes it most difficult for the cop since he will not be able to encroach the sides of the box further in than just three steps. If the robber moves in any other fashion at the beginning, then the same strategy can be used by the cop. It will be shown that the robber cannot escape the box if he is at a distance of five moves from any side of the box, there have been three walls built in each corner of the box, and it is the cop's turn as in Lemma 3.4.1.

Let T,B,L,R,C represent top, bottom, left, right, and corner, respectively, of the $35 \times 35$ box. The way the moves of the cop will be described should be interpreted as follows: two up from the BLC means that the bottom left corner is $(0,0)$ and two up from that would be $(0,2)$. We will play the first cop in the BLC and thus, we assume the robber is always moving diagonally toward the TRC but of course the strategy of the cop can be easily modified for the robber moving diagonally toward any corner. The robber by moving in the opposite direction of the second cop stops the cop from encroaching one or two of the sides by more than a total of three steps.

(1) The cop plays four up from the BLC.

(2) The cop plays two down from the TRC. 
(3) The cop plays one right of the TLC.

(4) The cop plays two right of the TLC.

(5) The cop plays four right of the TLC.

(6) The cop plays one down from the TRC.

(7) The cop plays four down from the TRC.

(8) It is now clear that the robber is moving to the TRC and thus, the cop can encroach the bottom side of the box in three steps to make it a $35 \times 32$ box by playing five up from the BLC.

(9) The cop plays seven up from the BLC.

(10) The cop plays three up and one left of the BRC.

(11) The cop plays three up and two left of the BRC.

(12) The cop plays three up and four left of the BRC.
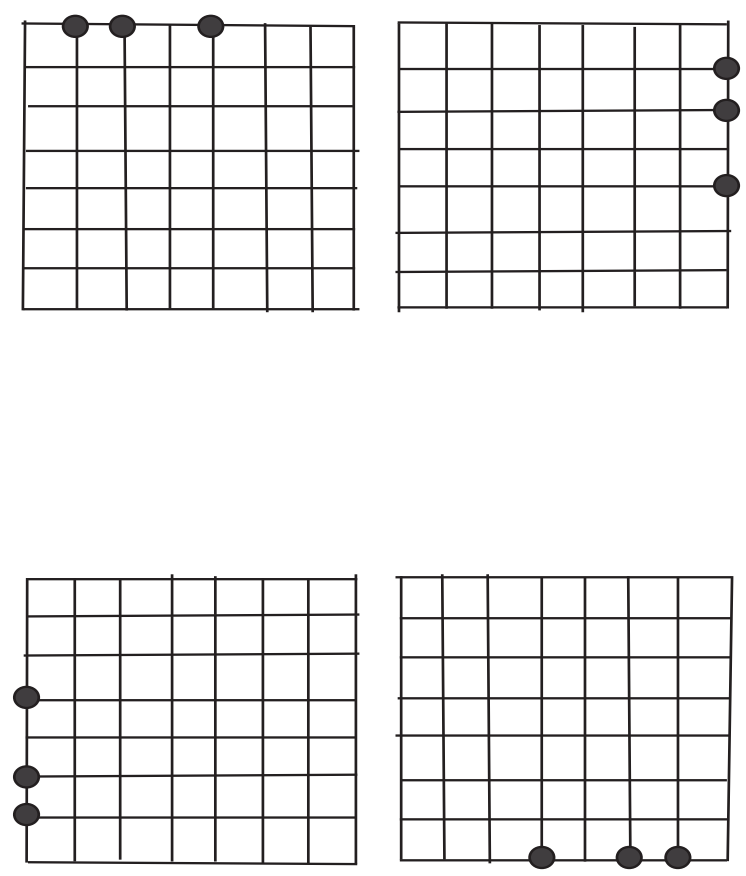

FigurE 3.11. Corners of the strong grid with the diagonal edges omitted. 
Now the next move the robber makes will bring him to within five moves of one or two sides. In the present case, the robber has moved to the TRC. If the robber moves up in any direction, then the cop's next move is to play on the side of the box directly above the robber. If the robber moves diagonally down and to the right or directly to the right then the cop's next move is to play on the side of the box directly to the right of the robber. From there in either case, we have the scenario where it is the robber's turn and he is distance five away from a side with a wall on the side directly in his path if he were to go straight at the side. We know that the cop can block the robber along this side now no matter which move he makes by Lemma 3.4.1 and Figure 3.12 below. With three walls built in each of the corners, the robber cannot run forever and must turn at the corners. We will assume the cop builds the entire box and that the robber can be anywhere inside that box afterwards. Therefore, it takes $35+35+30+30=130$ moves to build the walls of the box. 


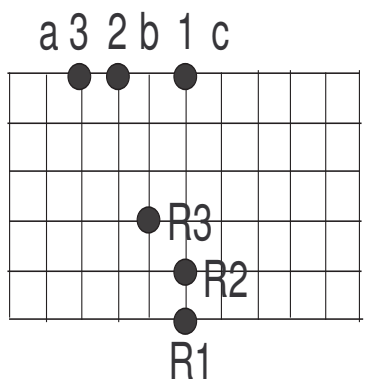

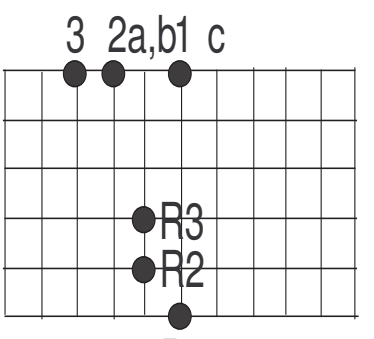

R1

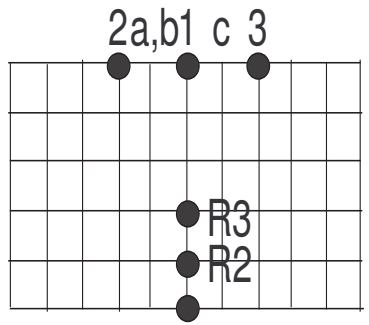

R1

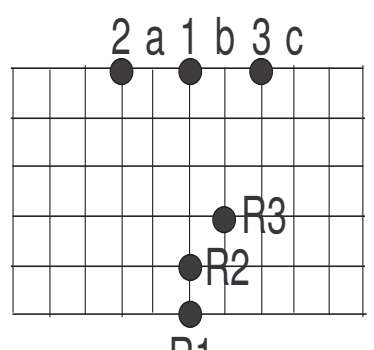

R1

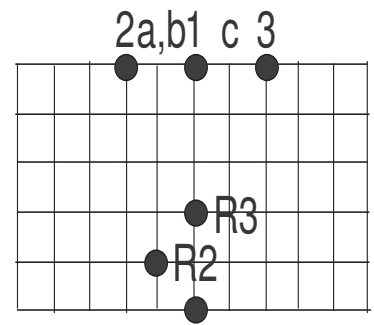

R1

Figure 3.12. Cop's strategy to stop robber reaching a side from a distance of five moves. In each of the figures, $a, b$, and $c$ represent the fourth wall the cop would build if the robber moved diagonally up and to the left or straight up or diagonally up and to the right, respectively. The final wall to build from there is obvious then.

We assume the robber is in the centre of the $35 \times 32$ box. The cop will now confine the robber to a $32 \times 12$ box. The two sides of length 12 have already been built as they are part of the sides of the $35 \times 32$ box. The cop will build the two sides of length 32 that are distance 11 from each other. The robber can only ever be distance five from either of these two sides but not both. This ensures the cop can stop the robber reaching either of these sides. 
It is difficult to know exactly what the best position the robber can attain while still making the cop complete the $32 \times 12$ box. Thus, we will say that once that box is completely built, we will let the robber be in the middle of this smaller box as that is best for him with the knowledge of the cop's strategy. Therefore, up to this point including the first move for the cop, there have been 131 moves for building the box and the first move and 60 moves for the two sides of length 32 in the smaller box for a total of 191 moves.

The robber is in the middle of the $32 \times 12$ box with the sides of this area completely covered with walls. The cop will now confine the robber to a $12 \times 12$ box. Two of the sides of length 12 have already been built as they are part of the sides of the $32 \times 12$ box. The cop will then build the other two sides distance 11 from each other with the robber directly in the middle so that he cannot be distance five from both of them at the same time. These two sides take 20 moves in total to build. Thus, the number of moves is now at 211.

We will again assume the robber to be anywhere in this new box which is $12 \times 12$ with the sides of this area completely covered with walls. The cop's strategy is to build a $6 \times 6$ box directly in the middle of the $12 \times 12$ box. This $6 \times 6$ box takes 20 moves to build. Now the robber can be inside the $6 \times 6$ box or outside it or he could have been on one of the 
vertices of the sides of the $6 \times 6$ box. Let these be cases $A$ through $\mathrm{C}$, respectively.

Case A: The robber is inside the $6 \times 6$ box.

The cop builds a $4 \times 4$ cross such that the intersection of the cross occurs in one of the middle vertices. The cop will build his first wall on the intersection vertex and it is guaranteed to be open as there are four possible intersection vertices in the $6 \times 6$ box. If the robber allows the cross to be built which means not being on one of those vertices while the cop needed to build a wall there, then he will be captured in at most three moves after that as a $2 \times 2$ area is the largest compartment left. This would total 10 moves for capturing him in the $6 \times 6$ box. If the robber occupies one of the vertices of the cross the cop needs to build a wall on, then the robber would occupy one of the vertices that is adjacent to the $2 \times 2$ compartment that will be left open. But then the cop just builds a wall adjacent to the robber in this $2 \times 2$ compartment. No matter what the robber does now, he will be captured in at most 10 moves. Therefore, Case A results in 10 moves.

Case B: The robber is outside the $6 \times 6$ box.

Since all the sides are symmetric we will consider the robber to be in the bottom $12 \times 3$ part of the $12 \times 12$ box. Then the cop can build two vertical walls or one horizontal and one vertical wall distance two and three away from the robber to confine him to a $7 \times 4$ box. We can say 
that it will take a maximum of 8 moves for the cop to capture the robber in that $7 \times 4$ area since there are only 10 open vertices in it as the cop can choose the two open vertices after the 8 moves such that they will not be adjacent. Therefore, Case B results in at most 12 moves.

Case C: The robber is on one of the vertices of one of the sides of the $6 \times 6$ box after all the other vertices of the sides of this box have a wall built on them.

If the robber is not on one of the corners of this $6 \times 6$ box, then the cop will build a wall on the outside of the $6 \times 6$ box on the adjacent vertex that is closest to a corner. The cop will keep playing adjacent to the robber on the outside of the $6 \times 6$ box until he cannot anymore or the robber moves. If the robber moves inside the $6 \times 6$ box then he will be captured in 10 moves by Case $A$. If he moves outside of the $6 \times 6$ box then he will be captured in 12 moves by Case B but the walls being built while he is on one of the sides of the box count towards these 12 moves. Therefore, if the robber does not occupy a corner vertex of the $6 \times 6$ box, then he will be captured in at most 13 moves by skipping his turn until he cannot move outside of the $6 \times 6$ box at which point he will move into the $6 \times 6$ box. This is due to there being exactly three adjacent vertices outside of the $6 \times 6$ box. Note that the move where the cop builds the wall to complete the $6 \times 6$ box was already counted before. 
If the robber is on one of the corners of the $6 \times 6$ box then there are five adjacent vertices outside of the $6 \times 6$ box. The cop will build a wall on each of these vertices and once he has done so the robber will move into the $6 \times 6$ box for the same reasons as above. This results in 15 moves for the cop. Therefore, Case $\mathrm{C}$ results in at most 15 moves.

Since Case C results in the most moves for the cop, then that would be the robber's strategy. Hence, we have proven the desired result that the total moves to capture the robber is $211+20+15=246$.

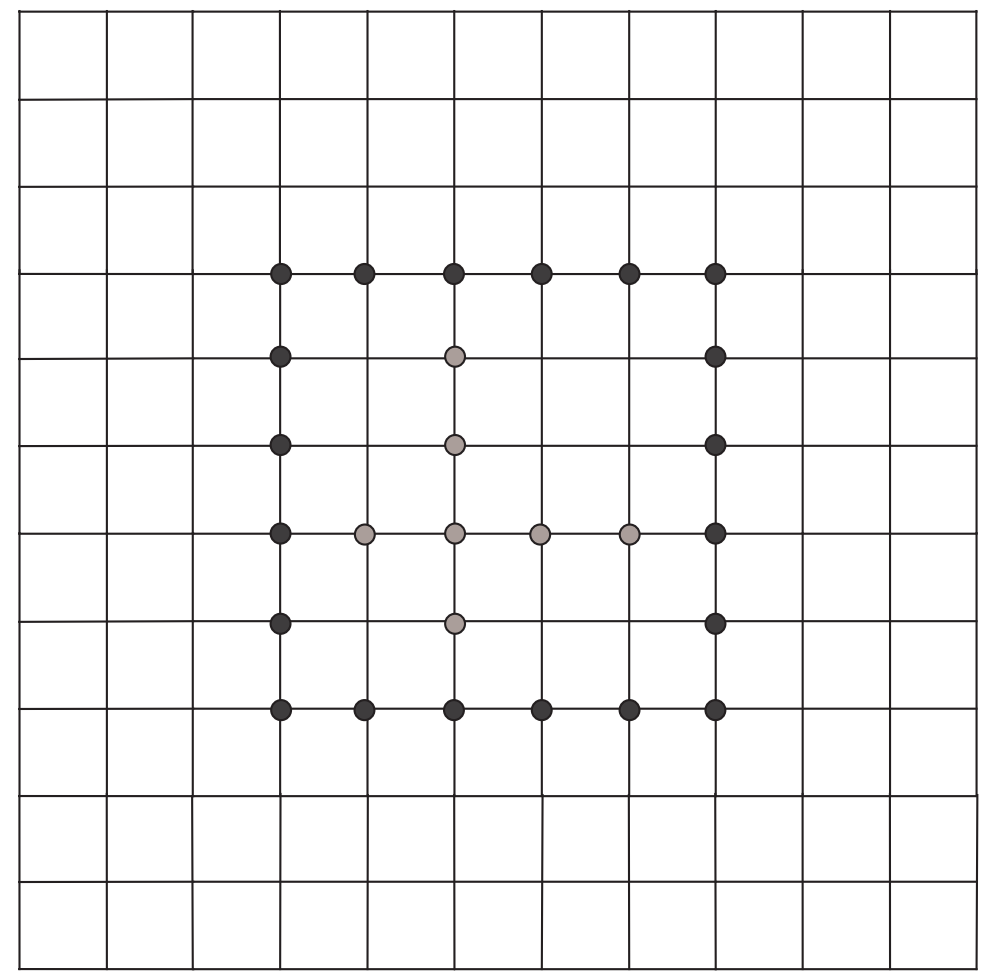

FiguRE 3.13. $12 \times 12$ box robber is trapped inside with the $6 \times 6$ box and $4 \times 4$ cross inside. 


\subsection{Wall Capture Time of an $n$-layered Infinite Cartesian Grid}

TheOREM 3.5.1. For $n$ a positive integer, we have that

$$
W_{c_{t}}\left(P_{\infty} \square P_{\infty} \square P_{n}\right) \leq 44 n^{2}+6 n+15 .
$$

PROOF. The cop will build an initial 2-dimensional box of dimensions $(8 n+3) \times(8 n+3)$ on each of the $n$ levels. The cop needs four corners built for each of the $n$ levels and the robber must be distance $4 n+1$ from each side for this, since he can be stopped at distance one from a side of the box if it is the cop's turn. Thus, the dimensions of the box needed are $2 \cdot(4 n+1)+1=8 n+3$. The cop can always encroach one of the sides one step in and one side $n$ steps in or the equivalent in combination of encroaching multiple sides based on the movement of the robber. This is possible since the cop builds one of the corners on each of the $n$ levels with the robber moving toward one of the two sides that corner does not touch. As soon as the cop starts building the second corner which will share the same $x$ or $y$ coordinate as the first corner, the robber must change direction so that he is going directly at the side that is opposite those two corners to reduce the amount the cop can encroach the last side. So $P_{\infty} \square P_{\infty} \square P_{n}$ really requires a $(8 n+3) \times(7 n+2)$ box. It takes $n \cdot(2 \cdot(8 n+1)+2 \cdot(7 n))=30 n^{2}+2 n$ moves to build the sides of the boxes on all levels. 


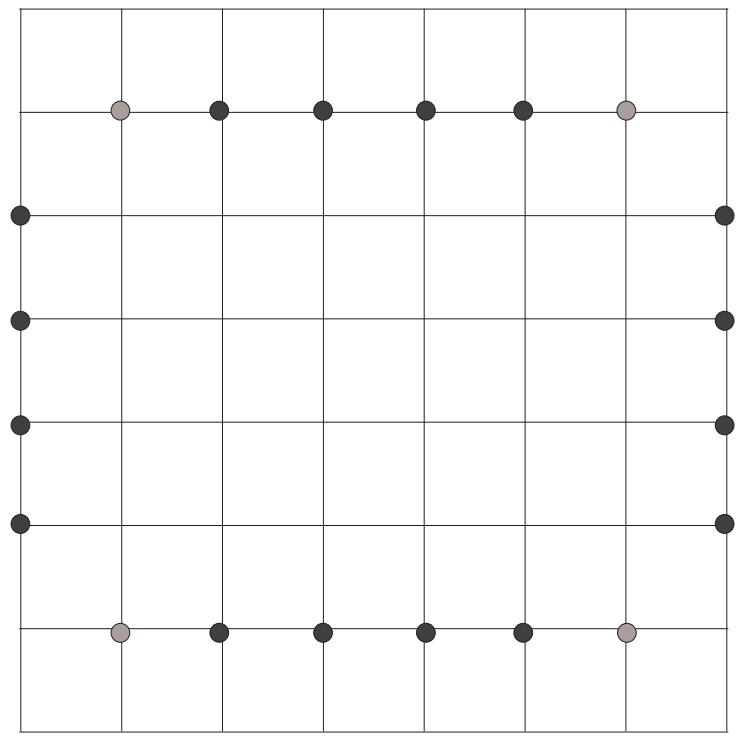

FiguRE 3.14. Representation with compressed dimensions of the $(8 n+$ $3) \times(7 n+2)$ box built on one of the $n$ levels. The grey vertices represent the corners built at the beginning.

Then we will assume that the robber is in the centre of the bottom level as that is the best possible position for him based on the cop's strategy. The cop will then build a side that is parallel to the $7 n+2$ side of the box that will be distance one away from the robber. The cop will also build another side that will be parallel to the previous side that will be distance two away from the robber's starting position. The robber cannot pass either of these sides as he can only ever be distance one away from either side with it being the cop's turn. Then it takes $2 n \cdot 7 n=14 n^{2}$ moves to build the sides on each level. Then, the robber will be in this new box that is $7 n \times 4$.

The cop will now make this new box into a $4 \times 4$ box containing the robber by building the two sides of length four that are perpendicular 
to the previous sides. The cop can build one of these sides distance one away from the robber and the other distance two away. Then it takes $4 n$ moves to build these sides on each level assuming again that the robber is in the best position possible.

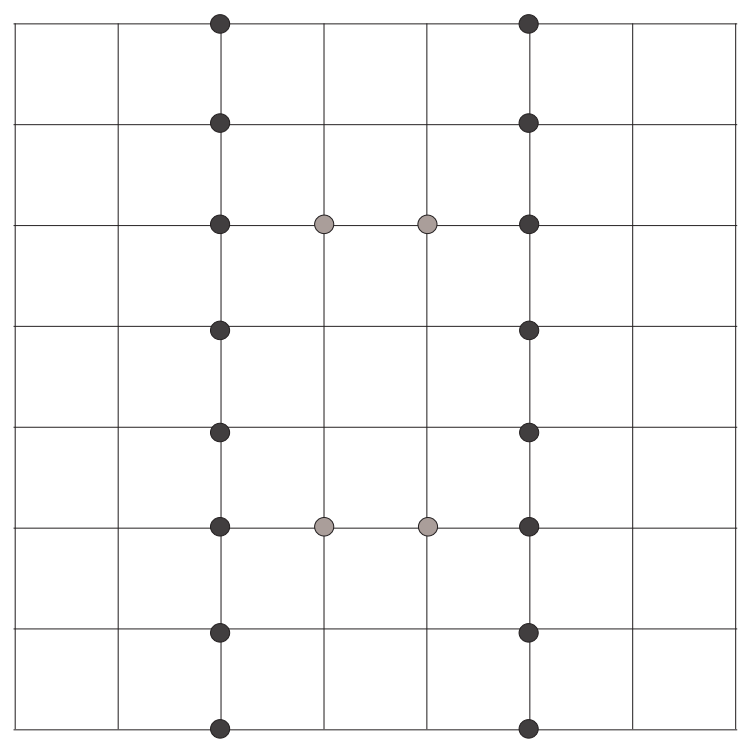

FiguRE 3.15. Smaller representation of $7 n \times 4$ box built on one of the $n$ levels with the $4 \times 4$ box built inside it.

The cop will then build a ceiling that is distance one above the robber and a floor that is distance two below the robber's starting position which again we assume is ideal. The ceiling and floor will take 8 moves to build and the robber will be confined to two levels of $2 \times 2$ open vertices or a $2 \times 2 \times 2$ cube of open vertices. From the example in Section 2.1, we know that such a cube has a wall capture time of 6 moves. Finally, it takes 14 moves to capture the robber inside these small $4 \times 4$ boxes. Also, we have the first move which is wasted and in total we have $44 n^{2}+6 n+15$ moves. 
CHAPTER 4

\section{Wall Cop Number}

\subsection{Introduction}

So far, we have studied graphs in which one cop is enough to capture the robber. Now, we will study graphs which may need more than one cop to ensure the capture of the robber. This involves the parameter of wall cop number.

Recall that the wall cop number of a graph $G$, written $W_{c}(G)$, is the least number of cops it takes for the cops to capture the robber in $G$ given that the cops and robber have played their best strategies. Note that every finite graph has wall cop number 1 .

We have already introduced several examples of graphs with a wall cop number equalling one as we have shown that these graphs have a finite wall capture time. Simple examples of graphs with wall cop number greater than one are harder to find as these graphs must be infinite which adds complexity. Therefore, as our first example, we will study $k$-regular trees which we discussed in Chapter 2. In Chapter 2, we considered finite $k$-regular trees. Here, we will consider infinite $k$-regular trees, written $T_{\infty, k}$ 


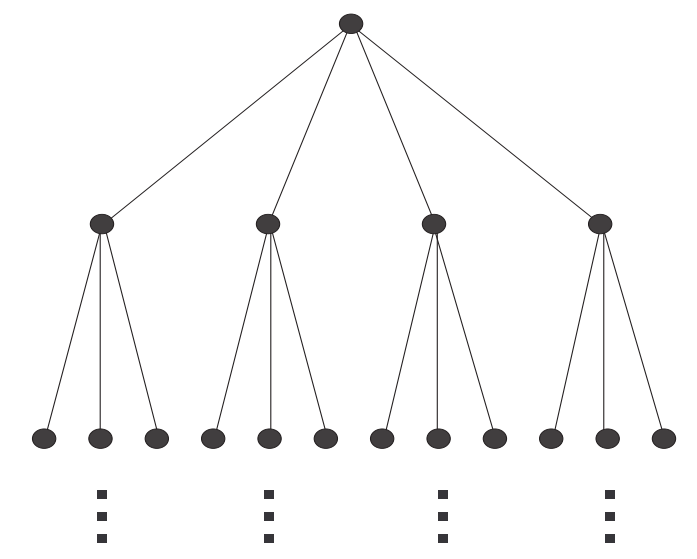

FiguRe 4.1. An infinite 4-regular tree.

THEOREM 4.1.1. $W_{c}\left(T_{\infty, k}\right)=k-1$ for infinite $k$-regular trees.

Proof. Recall, Theorem 2.4.2 which stated that the

$$
W_{c_{t}}\left(T_{k}\right)=(k-2) h\left(T_{k}\right)-k+5
$$

for finite $k$-regular trees with $h\left(T_{k}\right) \geq 3$ and $k \geq 3$.

This theorem shows that the wall capture time goes to infinity as the height of the tree goes to infinity. Therefore, $W_{c}\left(T_{\infty, k}\right)>1$. From the proof of Theorem 2.4.2, we know there is no strategy for one cop to capture the robber before he reaches the bottom of the tree. When considering the infinite $k$-regular tree, the cops must be able to confine the robber to a finite subgraph. This can only be done by forming a barrier of walls across a level of the tree to stop the robber continuously moving down the tree. From the proof of Theorem 2.4.2, we know this is not possible unless we have exactly $k-1$ cops as all vertices on the level 
below the robber must be blocked in order to stop him moving down to the next level.

The strategy for $k-1$ cops is to build walls on the $k-1$ adjacent vertices on the level below the robber. There will be exactly $k-1$ of these vertices as the cops will build a wall on the topmost vertex of the tree on their first turn. The robber will then have to move up the tree towards the topmost vertex. The cops build a wall on the $k-1$ vertices the robber is adjacent to, excluding the vertex he just came from. The robber must skip his turn and is captured on the next turn. Therefore, $W_{c}\left(T_{\infty, k}\right)=k-1$

Wall cop number is monotonic relative to subgraphs. This result gives lower bounds for the wall cop number of graphs if the wall cop number of one of their subgraphs is known. Also, if the wall cop number of a graph is known, then this result gives upper bounds for the wall cop number of subgraphs of that graph.

THEOREM 4.1.2. If $H$ is a subgraph of $G$, then $W_{c}(H) \leq W_{c}(G)$.

Proof. The cops play two games simultaneously: one in $G$ using their winning strategy there, and one in $H$. The robber is confined to $H$, and we view moves in $H$ by the robber as moves in $G$. The cops adapt their strategy in $G$ to one in $H$ as follows. If the cops build a wall in $G$ on a vertex $u$ also in $H$, then they build a wall on $u$ in the game on $H$. 
Each time the cops build a wall in $V(G) \backslash V(H)$, they can just choose any vertex without a wall in $H$, and build a wall there.

The adapted strategy in $H$ is winning for the cops, as in each round, the vertices without walls available to the robber is a subset of vertices without walls in $G$.

\subsection{Wall Cop Number of an Infinite-layered Infinite Hexagonal grid}

An infinite-layered infinite hexagonal grid, written $H_{\infty, \infty}$, is an infinitelayered perfect matching between two copies of $H_{\infty}$. Each layer is a copy of $H_{\infty}$ and each vertex in each copy of $H_{\infty}$ is adjacent to its corresponding vertex in the layer above and below. The labelling of the coordinates of the 3-D grid will follow the rule that the distance between any two adjacent vertices on the $x$-axis or $y$-axis or $z$-axis is one unit.

TheOREM 4.2.1. $W_{c}\left(H_{\infty, \infty}\right) \leq 2$.

PROOF. We will show a strategy for two cops to win, thereby proving an upper bound of two for the wall cop number. The cops' plan is to confine the robber to two layers. Note that since we are only dealing with wall cop number here, the number of moves it takes for the cops to capture the robber is irrelevant. Therefore, the robber will not skip his turn as no vertex has an infinite degree which would be the only case in which the robber would skip his turn. We will not mention the robber's 
ability to skip his turn below as we have shown it is irrelevant. We will now show the moves for the cops and robber. The moves can also be seen in Figure 4.2 below.

(1) C: The first move for the cops will be wasted as the grid is infinite.

$\mathbf{R}$ : The robber will place himself on the graph and we will denote this vertex as the origin, $(0,0,0)$. Without loss of generality, we will assume this vertex has an adjacent vertex at $(0,1,0)$.

(2) C: The cops play on $(-1,0,0)$ and $(1,0,0)$.

$\mathbf{R}$ : The robber will not move to $(0,1,0)$ as then the cops would play on $(-1,1,0)$ and $(1,1,0)$, forcing the robber to move up or down a layer except two more walls will have been built close to him. Therefore, the robber will move up a layer to $(0,0,1)$ as moving up or down a layer is symmetric.

(3) C: The cops play on $(0,0,2)$ and $(0,1,1)$.

$\mathbf{R}$ : The robber can only move left or right and since they are symmetric, the robber moves right to $(1,0,1)$.

(4) C: The cops play on $(1,0,2)$ and $(1,-1,1)$.

$\mathbf{R}$ : The robber can only move right to $(2,0,1)$.

(5) C: The cops play on $(2,0,2)$ and $(3,0,1)$.

$\mathbf{R}$ : The robber will not move down a layer to $(2,0,0)$ as then the cops would play on $(2,0,-1)$ and $(3,0,0)$ which would put the robber in the same case he is in now except more walls will have 
been built close to him. Therefore, the robber must move to $(2,1,1)$.

(6) C: The cops play on $(2,1,2)$ and $(3,1,1)$.

$\mathbf{R}$ : The robber will not move down a layer for similar reasons to the last turn. Therefore, the robber must move left to $(1,1,1)$.

(7) $\mathbf{C}$ : The cops play on $(1,1,2)$ and $(1,2,1)$.

$\mathbf{R}$ : The robber can only move down a layer to $(1,1,0)$.

(8) C: The cops play on $(1,1,-1)$ and $(1,2,0)$.

R: The robber will not move left as the cops would play adjacent on his left and on the layer below him, forcing him to move back to $(0,0,0)$ on his next turn. The cops would then play adjacent on the layers above and below him and he would have to skip his turn and be captured on the next turn. Therefore, the robber moves right to $(2,1,0)$.

(9) $\mathbf{C}$ : The cops play on $(2,1,-1)$ and $(3,1,0)$.

$\mathbf{R}$ : The robber will not move up a layer to $(2,1,1)$ as then the cops would play adjacent on his left and below him on the same layer, forcing him to skip his turn and be captured on the next turn. Thus, the robber will move down to $(2,0,0)$.

(10) C: The cops play on $(2,0,-1)$ and $(3,0,0)$.

$\mathbf{R}$ : The robber can only move up a layer to $(2,0,1)$.

(11) C: The cops play on $(2,1,1)$ and $(1,0,1)$. 
$\mathbf{R}$ : The robber must skip his turn.

(12) C: One of the cops plays on $(2,0,0)$ and the robber is captured.

Thus, we have shown that two cops suffice to capture the robber on $H_{\infty, \infty}$. Therefore, $W_{c}\left(H_{\infty, \infty}\right) \leq 2$. 
$z=2$

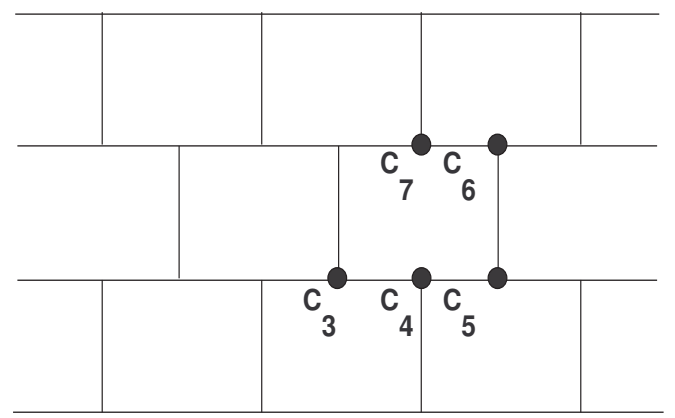

$z=1$

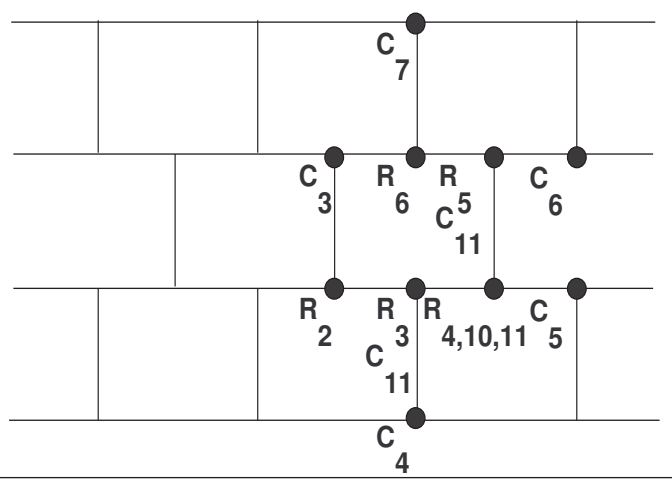

$\mathbf{z}=0$

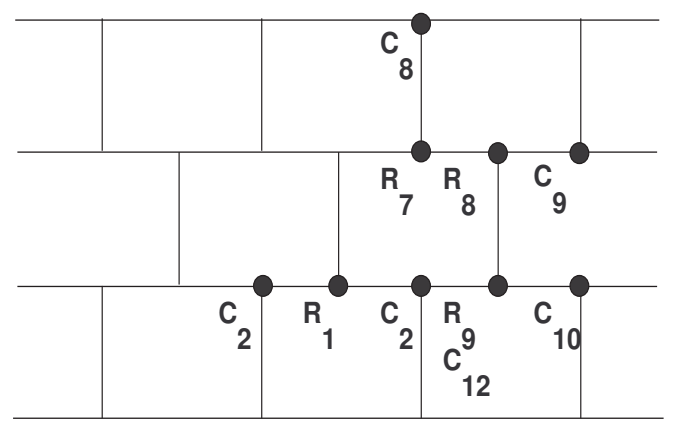

$z=-1$

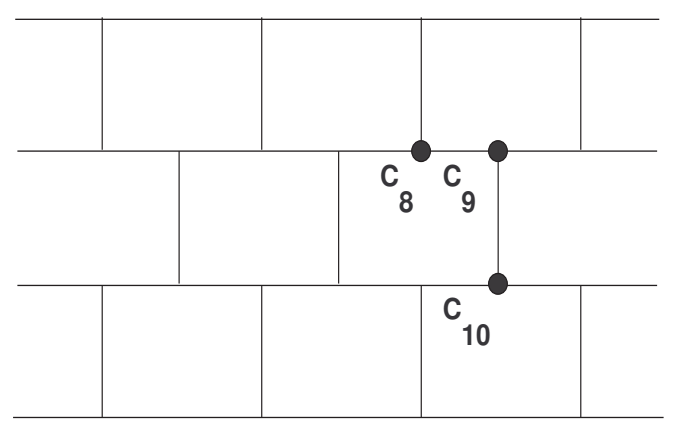

FiguRe 4.2. Cops and robber's moves in $H_{\infty_{\infty}}$. The labels correspond to the player's turn number and location on the closest vertex up and to the right. When both a cop label and a robber label are needed, the cop label appears below the robber labsel. $R_{4,10,11}$ means that the robber's $4^{\text {th }}, 10^{\text {th }}$, and $11^{\text {th }}$ moves are on that vertex. The cops' first move $C_{1}$ is not shown in the figure as it is sufficiently far away from these vertices. 


\subsection{Wall Cop Number of an Infinite-layered Infinite Cartesian grid}

An infinite-layered infinite Cartesian grid, written $P_{\infty} \square P_{\infty} \square P_{\infty}$, is the Cartesian products of three infinite, two-way paths. We will be labelling the vertices using Cartesian coordinates.

Theorem 4.3.1. $W_{c}\left(P_{\infty} \square P_{\infty} \square P_{\infty}\right) \leq 2$.

Proof. We will show a strategy for two cops to win, thereby proving an upper bound of two for the wall cop number. The cops' plan is to confine the robber to an $11 \times 11 \times 11$ cube. The robber's initial vertex will be at the centre of this cube. The first five moves for the cops will build walls near the eight corners of the cube with the first move wasted as the grid is infinite. The exact coordinates of these vertices as can be seen in Figure 4.3 are $(-4,-4,-5),(-4,4,-5),(4,-4,-5),(4,4,-5),(-4,-4,5)$, $(-4,4,5),(4,-4,5),(4,4,5)$. Now the robber is at least distance one away from one of the six sides of the cube since he starts at distance five away from each of the sides and it is the cops' turn. 


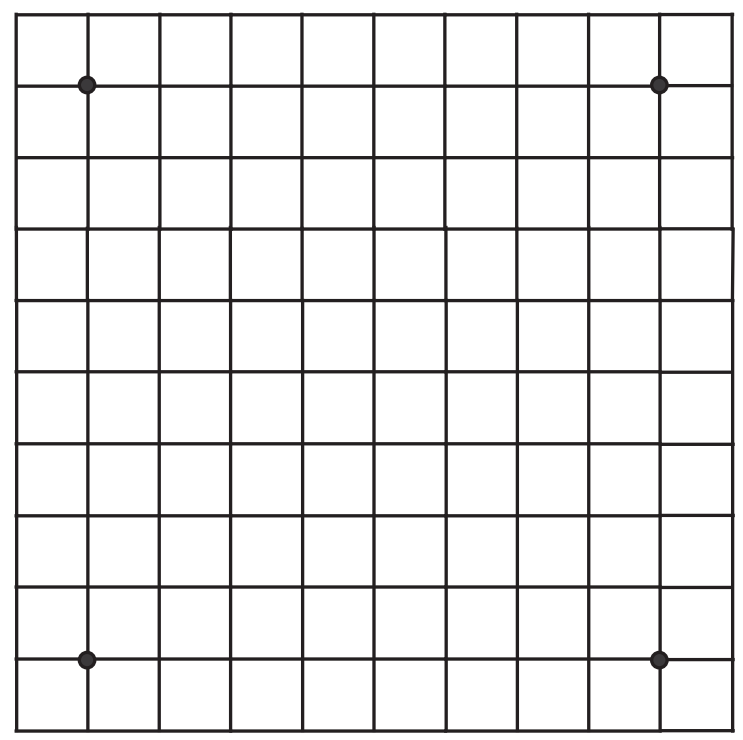

Figure 4.3. Fifth layer of the cube ( $x y$ plane at $z=5$ ). Cops' first moves are represented by the four vertices in the corners. The figure is the same for the $z=-5$ layer of the cube.

When the robber resides in the top layer or bottom layer of the cube, one of the cops will keep him confined in the two-dimensional plane. This is possible since in the top and bottom layer of the cube, the robber can only ever be adjacent to one of the sides of the cube. The other cop will play directly above the robber on the layer above him if the robber is in the top layer or directly below him if he is in the bottom layer.

When the robber resides in any of the other layers of the cube, both cops will work to keep him confined in the two-dimensional plane. This is possible since in these layers of the cube, the robber can only ever be adjacent to two sides of the cube. 
Thus, the robber can never escape this cube. Since the cube is finite, the robber will eventually be captured. Therefore, $W_{c}\left(P_{\infty} \square P_{\infty} \square P_{\infty}\right) \leq 2$.

\subsection{Wall Cop Number of Layered Strong Grids}

An infinite-layered infinite strong grid, written $P_{\infty} \otimes P_{\infty} \otimes P_{\infty}$, is the strong products of three infinite, two-way paths. We will be labelling the vertices using Cartesian coordinates.

THEOREM 4.4.1. For $n$ a positive integer with $n \leq 14$, we have that

$$
W_{c}\left(P_{\infty} \otimes P_{\infty} \otimes P_{n}\right) \leq n .
$$

Proof. This proof uses the proof in Chapter 3 of how the one cop wins on a strong grid. When we have $n$ layers of strong grids, we employ one cop per layer for a total of $n$ cops, all using the same strategies but on their respective layers. They all play the same moves on their respective layers and they all employ the strategy to win on their layer which was introduced in the strong grid proof. Thus, it only requires one cop per layer, so $W_{c}\left(P_{\infty} \otimes P_{\infty} \otimes P_{n}\right) \leq n$ for $n \leq 14$.

TheOREM 4.4.2. $W_{c}\left(P_{\infty} \otimes P_{\infty} \otimes P_{\infty}\right) \leq 14$.

Proof. (1) C: The first move for the cops is wasted since the graph is infinite. 
$\mathbf{R}$ : The robber plays sufficiently far away from the walls built on the cops' first moves and we will call this the origin $(0,0,0)$.

(2) C: The first 12 cops build walls to form four columns of three walls all diagonal to the robber: $(1,1,1),(1,1,0),(1,1,-1),(1,-1,1),(1,-1,0)$, $(1,-1,-1),(-1,1,1),(-1,1,0),(-1,1,-1),(-1,-1,1),(-1,-1,0),(-1,-1,-1)$. The last two cops play on one side of the robber: $(-1,0,0),(-1,0,-1)$. See Figure 4.4.

$\mathbf{R}$ : The robber will move to $(1,0,1)$ as this is away from the most walls.

(3) C: The cops play on $(2,0,0),(2,0,1),(2,0,2),(2,-1,0),(2,-1,1)$, $(2,-1,2),(2,1,0),(2,1,1),(2,1,2),(1,-1,2),(1,1,2),(0,-1,2),(0,0,2),(0,1,2)$. See Figure 4.5 and Figure 4.6.

$\mathbf{R}$ : The robber wants to move away from any walls already built. He will not move up to $(1,0,2)$ as otherwise he will be captured next turn since he would be adjacent to 13 walls and the cops can build 14 more. Therefore, the robber moves to $(0,-1,0)$ as here he will only be adjacent to eight walls already built which is the fewest. Also, this moves away from all walls built above him on the previous turn. See Figure 4.7.

(4) C: The cops play on $(-1,-2,-1),(-1,-2,0),(-1,-2,1),(0,-2,-1),(0,-2,0)$, $(0,-2,1),(1,-2,-1),(1,-2,0),(1,-2,1),(0,0,-1),(0,0,0),(0,0,1),(-1,0,1)$, $(0,-1,-1)$. See Figure 4.8, Figure 4.9, and Figure 4.10. 
$\mathbf{R}$ : The robber can only move to $(1,0,-1)$ or $(1,0,0)$. Thus, the robber moves to $(1,0,-1)$ as it is adjacent to 10 walls which is the fewest. See Figure 4.11.

(5) C: The cops will force the robber to move directly up to $(1,0,0)$ by playing on $(0,-1,-2),(0,0,-2),(0,1,-2),(1,-1,-2),(1,0,-2),(1,1,-2)$, $(2,-1,-2),(2,0,-2),(2,1,-2),(2,-1,-1),(2,0,-1),(2,1,-1),(0,1,-1),(0,1,0)$. See Figure 4.12 and Figure 4.13.

$\mathbf{R}$ : The robber moves up to $(1,0,0)$.

(6) C: The cops play on $(0,1,1),(0,-1,0),(1,0,-1),(1,0,1)$ and the robber is captured.

Therefore, 14 cops suffice to capture the robber on $\left(P_{\infty} \otimes P_{\infty} \otimes P_{\infty}\right)$. Thus, $W_{c}\left(P_{\infty} \otimes P_{\infty} \otimes P_{\infty}\right) \leq 14$.

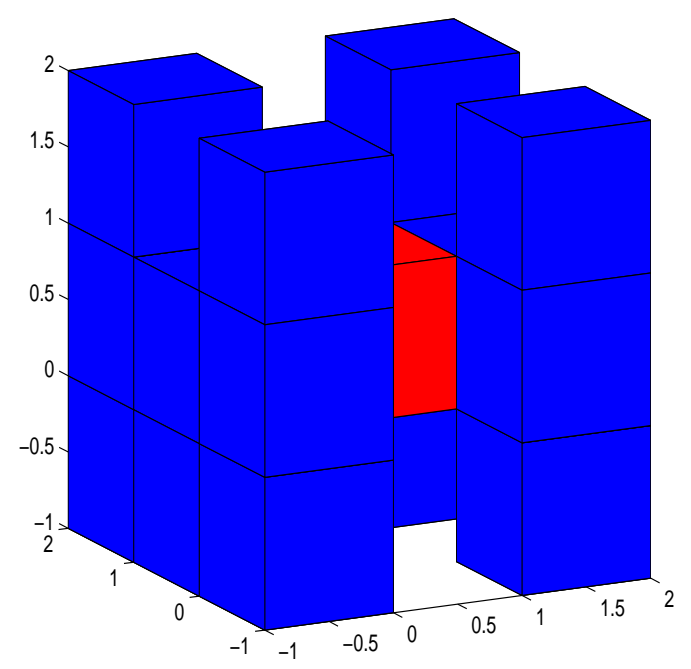

Figure 4.4. Robber's position in red after $2^{\text {nd }}$ turn for cops in blue. 


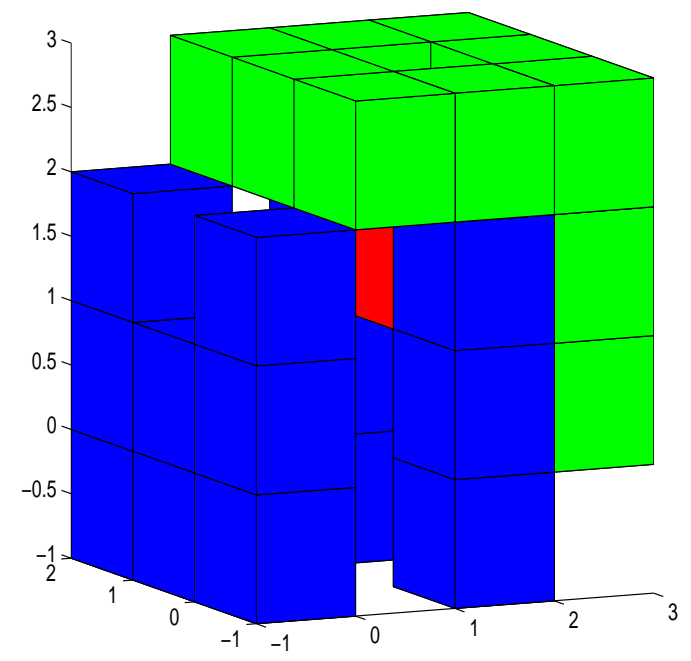

FiguRE 4.5. Robber's position in red after $3^{\text {rd }}$ turn for cops in green.

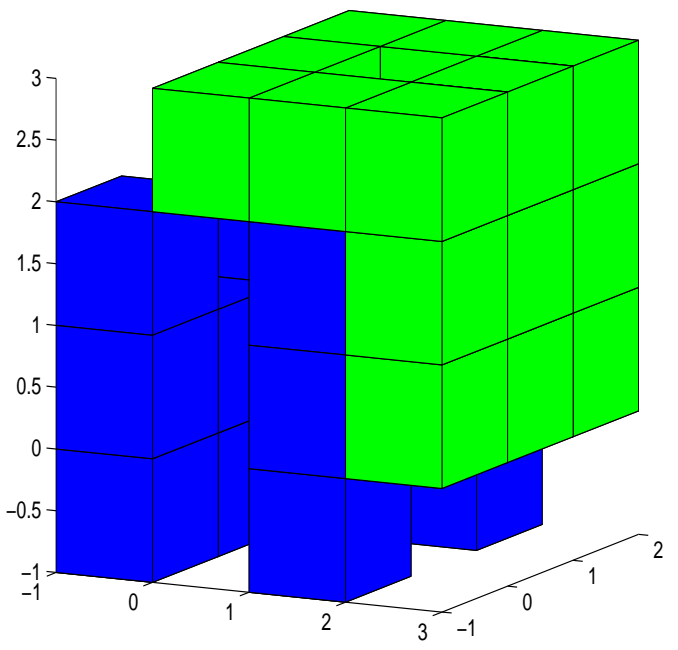

FiguRE 4.6. Robber's position in red after $3^{\text {rd }}$ turn for cops in green. 


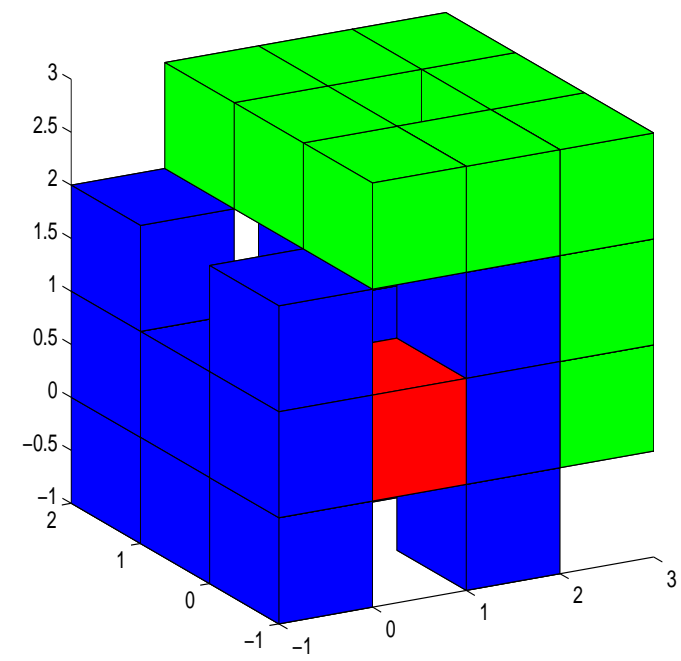

FiguRE 4.7. Robber's position in red before $4^{\text {th }}$ turn for cops.

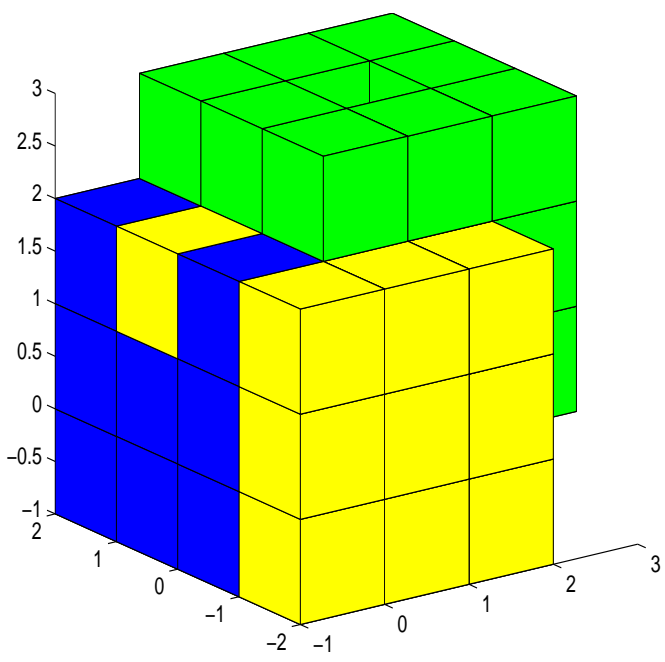

FIgURE 4.8. Robber's position in red at $(0,-1,0)$ (cannot be seen) after $4^{\text {th }}$ turn for cops in yellow. 


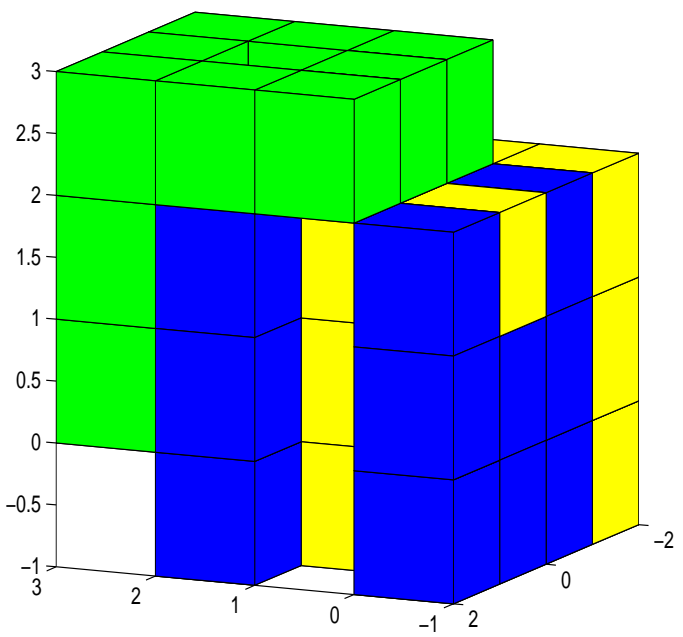

Figure 4.9. Robber's position in red at $(0,-1,0)$ (cannot be seen) after $4^{\text {th }}$ turn for cops in yellow.

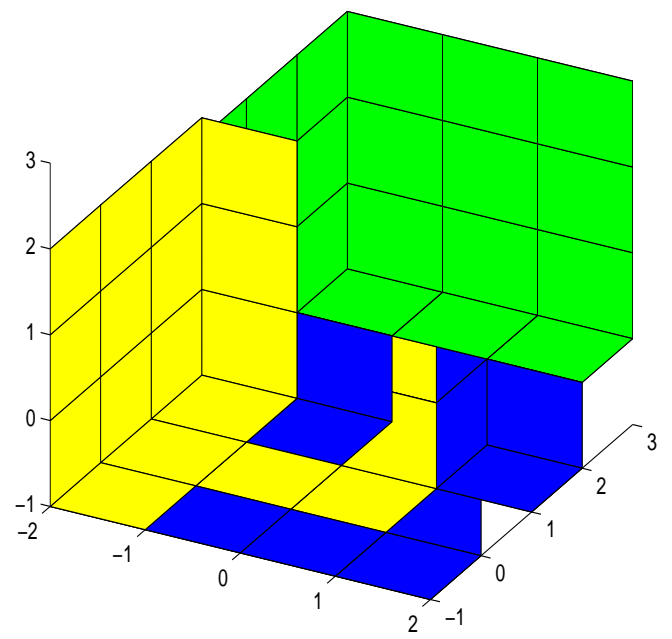

FiguRE 4.10. Robber's position in red at $(0,-1,0)$ (cannot be seen) after $4^{\text {th }}$ turn for cops in yellow. 


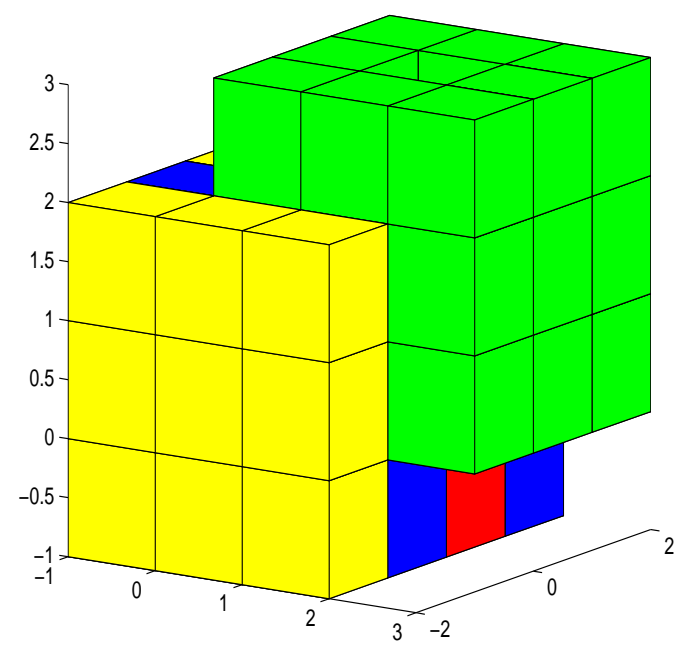

FIGURE 4.11. Robber's position in red before $5^{\text {th }}$ turn for cops.

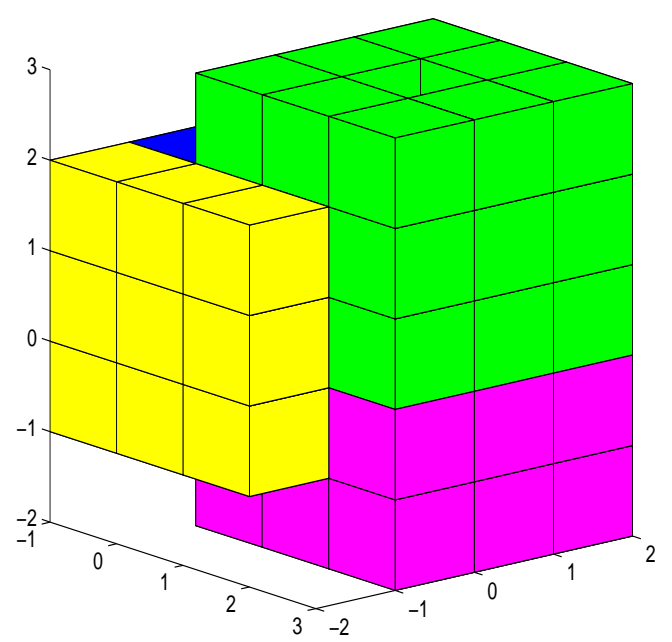

FiguRE 4.12. Robber's position in red (cannot be seen) after $5^{\text {th }}$ turn for cops in magenta. 


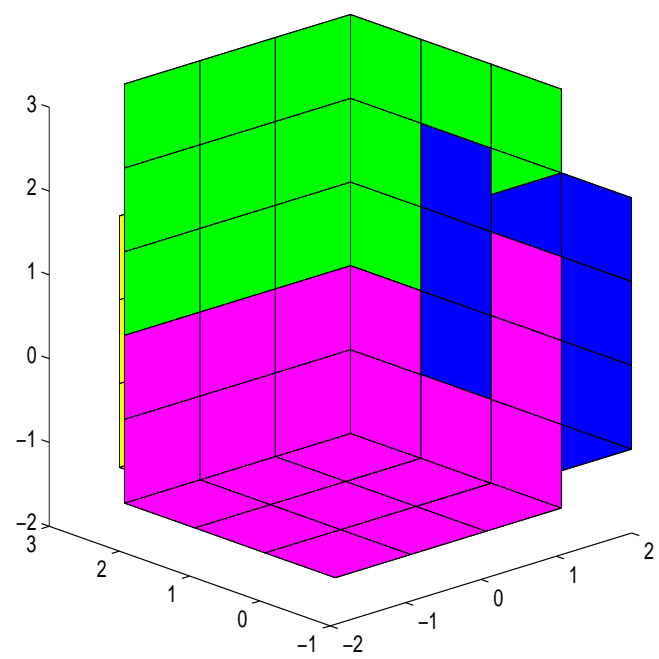

Figure 4.13. Robber's position in red (cannot be seen) after $5^{\text {th }}$ turn for cops in magenta.

COROLlary 4.4.3. For $n$ a positive integer with $n \geq 15$, we have that

$$
W_{c}\left(P_{\infty} \otimes P_{\infty} \otimes P_{n}\right) \leq 14 .
$$

Proof. The proof follows by Theorem 4.4.1 and Theorem 4.4.2. 


\section{CHAPTER 5}

\section{Wall Cops and Wall Robbers}

\subsection{Introduction}

In this chapter, we will study a variation of the game of Wall Cops and Robbers called Wall Cops and Wall Robbers. The game is played the exact same way as Wall Cops and Robbers except for the robber also builds walls now. The robber builds a wall on each vertex he occupies. These walls do not affect the cops in any way but they hinder the robber himself. The walls the robber builds have the same properties as the ones the cops build and therefore, the robber may never revisit a vertex he has previously occupied. In addition, the robber may not skip his turn.

The wall robber capture time of a graph $G$, written $W_{c_{t}}^{R}(G)$, is the least number of moves it takes for the cop to capture the wall robber in $G$ given that the cop and robber have both played their best strategies. The wall robber cop number of a graph $G$, written $W_{c}^{R}(G)$, is the least number of cops it takes for the cops to capture the wall robber in $G$ given that the cops and robber have played their best strategies.

We will study the wall robber capture time of the infinite hexagonal grid, written $H_{\infty}$, and the wall robber capture time of the infinite Cartesian grid, written $P_{\infty} \square P_{\infty}$. 
THEOREM 5.1.1. (1) $W_{c_{t}}^{R}(H) \leq W_{c_{t}}^{R}(G)$ if $H$ is a subgraph of $G$.

(2) $W_{c}^{R}(H) \leq W_{c}^{R}(G)$ if $H$ is a subgraph of $G$.

(3) $W_{c_{t}}^{R}(G) \leq W_{c_{t}}(G)$

(4) $W_{c}^{R}(G) \leq W_{c}(G)$

Proof. To prove (1) and (2), consider the cops as playing two games simultaneously: one in $G$ using their winning strategy there, and one in $H$. The robber is confined to $H$, and we view moves in $H$ by the robber as moves in $G$. The cops adapt their strategy in $G$ to one in $H$ as follows. If the cops build a wall in $G$ on a vertex $u$ also in $H$, then they build a wall on $u$ in the game on $H$. Each time the cops build a wall in $V(G) \backslash V(H)$, they can just choose any vertex without a wall in $H$, and build a wall there.

The adapted strategy in $H$ is winning for the cops, as in each round, the vertices without walls available to the robber is a subset of vertices without walls in $G$. Hence, the robber will be captured in $W_{c_{t}}^{R}(G)$-moves or fewer in $H$ if one cop is playing. Otherwise, if multiple cops are playing, then the number of cops required to capture the robber in $G$ will suffice to capture him in $H$.

To prove (3) and (4), consider that since the robber is also building walls, the number of possible moves for both players is less or equal than in the original game. Hence, the game of Wall Cops and Wall Robbers 
is never longer or needing more cops than the game of Wall Cops and Robbers when played on the same graph.

\subsection{Wall Robber Capture Time of an Infinite Hexagonal Grid}

Recall from Chapter 3, that the infinite hexagonal grid, written $H_{\infty}$, is a tiling of the plane by hexagons with vertices represented as vertices of the hexagons. The labelling of the coordinates of the grid will follow the rule as in Chapter 3, that the distance between any two adjacent vertices on the $x$-axis or $y$-axis is one unit.

THEOREM 5.2.1. $W_{c_{t}}^{R}\left(H_{\infty}\right)=6$.

Proof. We first provide a strategy for the cop which will take six moves to capture the robber. We will then describe why no fewer than six moves suffice to capture the robber.

(1) The first move of the cop is wasted since the grid is infinite. The robber plays sufficiently far away from that first wall rendering it useless. Due to symmetry, we can assume that the robber is on a vertex that has two adjacent open vertices, one on its left and one on its right, and one adjacent open vertex above it. This vertex will be the origin $(0,0)$.

(2) The cop plays directly adjacent to the left of the robber on $(-1,0)$. The robber can either move up or right and as they are symmetric, the robber moves right to $(1,0)$. 
(3) The cop plays to the right of the robber on $(2,0)$. The robber must move down to $(1,-1)$.

(4) The cop plays to the right of the robber on $(2,-1)$. The robber must move left to $(0,-1)$.

(5) The cop plays below the robber on $(0,-2)$. The robber must move left to $(-1,-1)$.

(6) The cop plays to the left of the robber on $(-2,-1)$ and the robber is captured in a total of six moves.

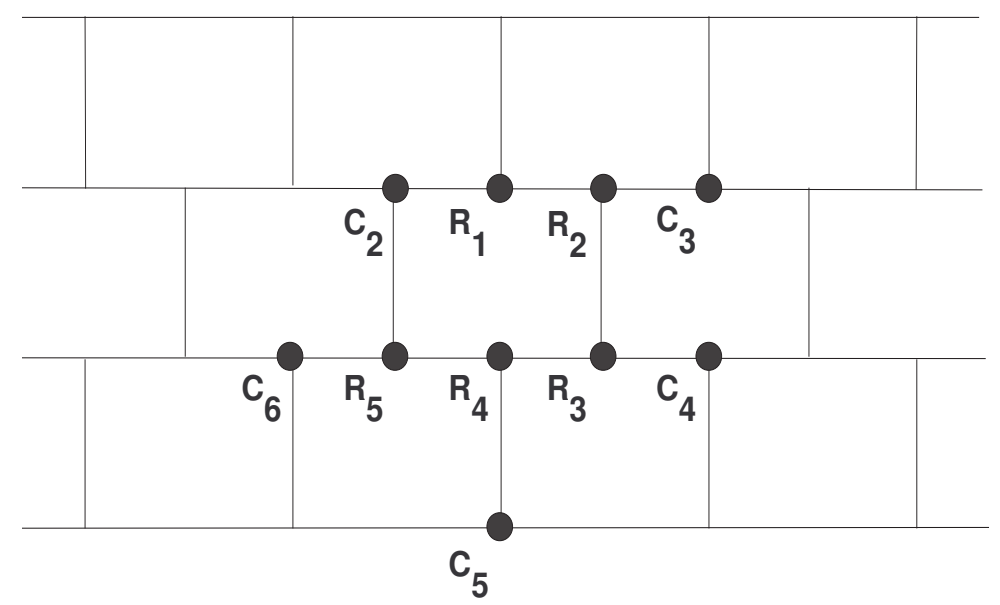

Figure 5.1. Cop and robber's moves. The labels correspond to the player's turn number and location on the closest vertex up and to the right. The cop's first move $C_{1}$ is not shown in the figure as it is sufficiently far away from these vertices.

This is the best strategy for the cop as the only other option for cop moves would be to play not adjacent to the robber but further away. If this is done at any stage, the robber will just treat it as if the cop had played adjacent to him in that direction so he will not move in the 
direction of this cop and following his strategy above, he will guarantee at least six moves.

\subsection{Wall Robber Capture Time of an Infinite Cartesian Grid}

Recall from Chapter 3, that the infinite Cartesian grid, written $P_{\infty} \square P_{\infty}$, is the Cartesian product of two infinite, two-way paths. We will be labelling the vertices using Cartesian coordinates.

TheOrem 5.3.1. $W_{c_{t}}^{R}\left(P_{\infty} \square P_{\infty}\right) \leq 10$

PROOF. The cop's plan is to trap the robber in a subgraph in which he will then capture the robber. The first move by the cop will be wasted as the grid is infinite and so the robber will play sufficiently far away from that first wall rendering it useless. The first vertex the robber plays on will be denoted as the origin $(0,0)$. The second move for the cop is to play adjacent to the robber and due to symmetry, it does not matter which vertex the cop chooses so the cop will play to the right of the robber on $(1,0)$. Now the robber has two choices since moving up and down are symmetric so we will divide this into cases with Case 1 being that the robber moves up to $(0,1)$ and Case 2 being that the robber moves left to $(-1,0)$.

Case 1: The robber moved up to $(0,1)$. The cop plays on $(1,2)$. Now the robber has three choices. If he moves to the right, then he will be captured next turn as that is moving into a trap. Thus, he can move left 
or up so we will divide this into cases with Case 1.1 being that he moved up to $(0,2)$ and Case 1.2 being that he moved left to $(-1,1)$.

Case 1.1: The robber moved up to $(0,2)$. We will now start the count again for the moves of the cop and robber.

(1) C: The cop plays on $(0,3)$.

$\mathbf{R}$ : The robber can only move left to $(-1,2)$.

(2) C: The cop plays on $(-2,3)$.

$\mathbf{R}$ : If the robber moves up, then he will be captured next turn as he would be moving into a trap. If the robber moves down, then he will be captured in 8 moves as the cop would play on $(-2,0)$. Thus, the robber moves left to $(-2,2)$.

(3) C: The cop plays on $(-3,2)$.

$\mathbf{R}$ : The robber can only move down to $(-2,1)$.

(4) C: The cop plays on $(-3,0)$.

$\mathbf{R}$ : The robber moves down to $(-2,0)$ as his only other options are moving left into a trap or right, both which result in him being captured next turn.

(5) C: The cop plays on $(-2,-1)$.

$\mathbf{R}$ : The robber can only move right to $(-1,0)$.

(6) C: The cop plays on $(-1,-1)$.

$\mathbf{R}$ : The robber can only move up to $(-1,1)$. 
(7) C: The cop can play anywhere as he wins once it is the robber's turn as the robber is already captured.

The robber is captured in 10 moves and this concludes Case 1.1. 


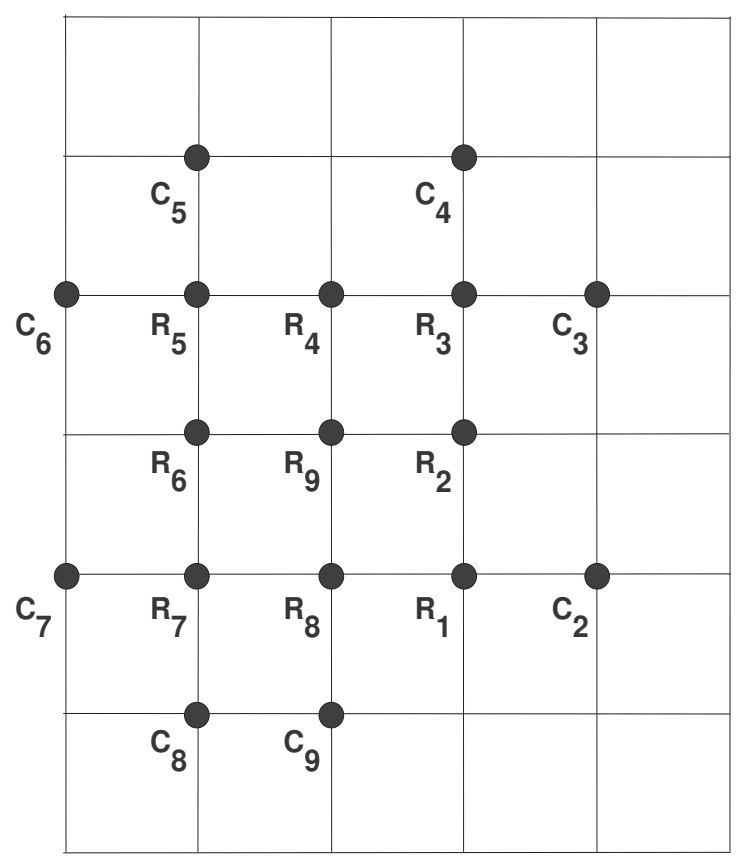

Figure 5.2. Cop and robber's moves in Case 1.1. The labels correspond to the player's turn number and location on the closest vertex up and to the right. The cop's first move $C_{1}$ is not shown in the figure as it is sufficiently far away from these vertices. The cop's tenth move $C_{10}$ is not shown in the figure as it is irrelevant in the capture of the robber.

Case 1.2: The robber moved left to $(-1,1)$. We will now start the count again for the moves of the cop and robber.

(1) C: The cop plays on $(-2,1)$.

$\mathbf{R}$ : If the robber moves down then the cop will play on $(-2,-1)$ and capture the robber in a total of 8 moves. Thus, the robber moves up to $(-1,2)$.

(2) C: The cop plays on $(-2,3)$.

$\mathbf{R}$ : If the robber moves left, he will be captured next turn as he would move into a trap. If the robber moves right, he will be 
captured next turn as the cop would play on $(0,3)$. Thus, the robber moves up to $(-1,3)$.

(3) C: The cop plays on $(-1,4)$.

R: The robber can only move right to $(0,3)$.

(4) C: The cop plays on $(1,4)$.

R: No matter where the robber moves, he will be captured next turn. Thus, the robber moves down to $(0,2)$.

(5) C: The cop can play anywhere as he wins once it is the robber's turn as the robber is already captured.

The robber is captured in 8 moves, thus, this is not an optimal strategy for the robber and this concludes Case 1.2. 


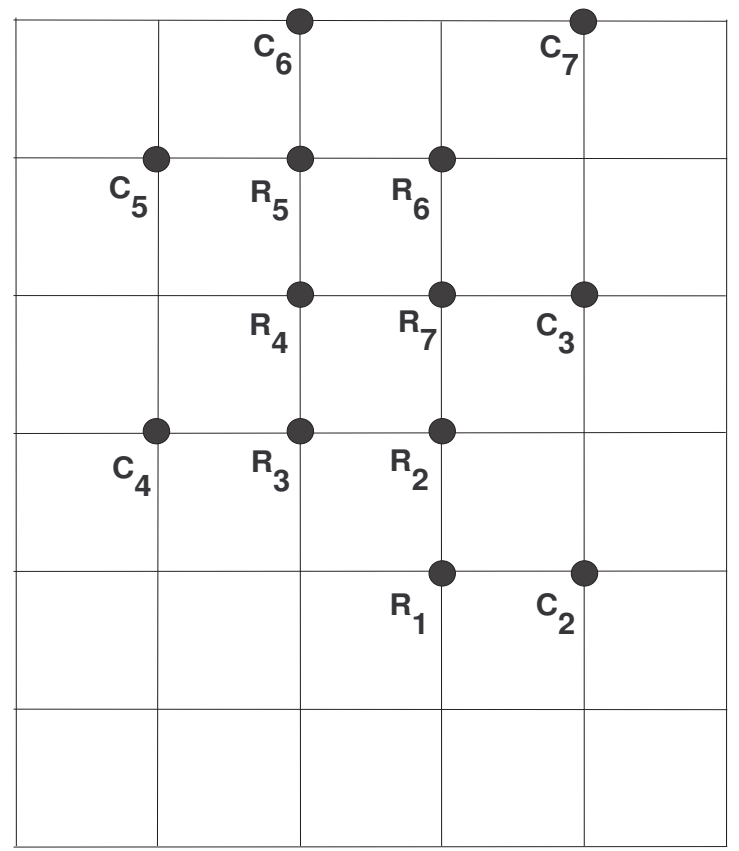

Figure 5.3. Cop and robber's moves in Case 1.2. The labels correspond to the player's turn number and location on the closest vertex up and to the right. The cop's first move $C_{1}$ is not shown in the figure as it is sufficiently far away from these vertices. The cop's tenth move $C_{8}$ is not shown in the figure as it is irrelevant in the capture of the robber.

Case 1.1 and Case 1.2 have been resolved and thus, this concludes Case 1.

Case 2: The robber moved left to $(-1,0)$. We will now start the count again for the moves of the cop and robber.

(1) C: The cop plays on $(-2,0)$.

$\mathbf{R}$ : The robber can only move up and down and as these are symmetric the robber moves up to $(-1,1)$.

(2) C: The cop plays on $(-2,2)$. 
$\mathbf{R}$ : If the robber moves left then he will be captured next turn as he would move into a trap. If the robber moves right then the cop would play on $(1,2)$ and the robber would be captured in a total of 7 moves. Thus, the robber moves up to $(-1,2)$.

(3) C: The cop plays on $(-1,3)$.

$\mathbf{R}$ : The robber can only move right to $(0,2)$.

(4) C: The cop plays on $(1,3)$.

$\mathbf{R}$ : If the robber moves down or up he will be captured next turn. Thus, the robber moves right to $(1,2)$.

(5) C: The cop plays on $(2,2)$.

$\mathbf{R}$ : The robber can only move down to $(1,1)$.

(6) C: The cop plays on $(2,1)$.

$\mathbf{R}$ : The robber can only move left to $(0,1)$.

(7) $\mathbf{C}$ : The cop can play anywhere as he wins once it is the robber's turn as the robber is already captured.

The robber is captured in 9 moves, thus, this is not an optimal strategy for the robber and this concludes Case 2. 


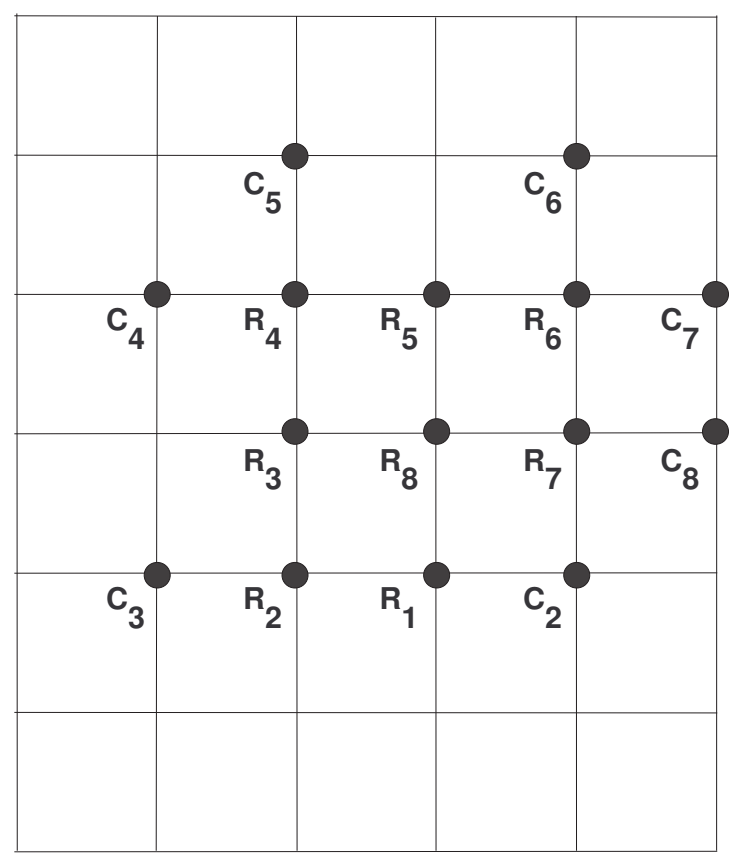

Figure 5.4. Cop and robber's moves in Case 2. The labels correspond to the player's turn number and location on the closest vertex up and to the right. The cop's first move $C_{1}$ is not shown in the figure as it is sufficiently far away from these vertices. The cop's tenth move $C_{9}$ is not shown in the figure as it is irrelevant in the capture of the robber.

All the cases have been shown to result in 10 moves or less for the cop to win. Therefore, $W_{c_{t}}^{R}\left(P_{\infty} \square P_{\infty}\right) \leq 10$. 


\section{CHAPTER 6}

\section{Conclusion and Open Problems}

In our final chapter, we summarize the results from the thesis and present some open problems collected from the entire thesis. Recall that we introduced the wall capture time of a graph $G$, written $W_{c_{t}}(G)$, as the least number of moves it takes for the cop to capture the robber in $G$ given that the cop and robber have both played their best strategies. We

also introduced that the wall cop number of a graph $G$, written $W_{c}(G)$, is the least number of cops it takes for the cops to capture the robber in $G$ given that the cops and robber have played their best strategies. Note that every finite graph has wall cop number 1 .

The table below gives a summary of the bounds on the above parameters that were found for the various graph classes we studied throughout the thesis. 


\begin{tabular}{|c|c|c|}
\hline Graph & $W_{c_{t}}$ & $W_{c}$ \\
\hline \hline$T_{k}$ with $h\left(T_{k}\right) \geq 3$ and $k \geq 3$ & $(k-2) h\left(T_{k}\right)-k+5$ & 1 \\
\hline$T_{\infty, k}$ & Undefined & $k-1$ \\
\hline$Q_{n}$ for $n \geq 1$ & $\leq 2^{n-1}+n-1$ & Open \\
\hline$H_{\infty}$ & 8 & 1 \\
\hline$H_{\infty, \infty}$ & Open & 1 \\
\hline$P_{\infty} \square P_{\infty}$ & $\leq 14$ & $\leq 2$ \\
\hline$P_{\infty} \square P_{\infty} \square P_{n}$ & $\leq 44 n^{2}+6 n+15$ & 1 \\
\hline$P_{\infty} \square P_{\infty} \square P_{\infty}$ & Open & 1 \\
\hline$\triangle_{\infty}$ & $\leq 138$ & $\leq n$ for $n \leq 14$ \\
\hline$P_{\infty} \otimes P_{\infty}$ & $\leq 246$ & $\leq 14$ for $n \geq 15$ \\
\hline$P_{\infty} \otimes P_{\infty} \otimes P_{n}$ & Open & $\leq 14$ \\
\hline$P_{\infty} \otimes P_{\infty} \otimes P_{n}$ & Open & Open \\
\hline$P_{\infty} \otimes P_{\infty} \otimes P_{\infty}$ & & \\
\hline
\end{tabular}

\subsection{Open Problems}

We will now present some open problems.

(1) Tight lower bounds.

For most of the graph classes we have studied, we only have an upper bound for the wall capture time or wall cop number. To improve on our results, one could find tight lower bounds for 
these two parameters. It is important that these lower bounds be tight in the sense that they equal the upper bounds.

(2) $W_{c_{t}}\left(P_{\infty} \square P_{\infty} \square P_{\infty}\right)$.

This graph is the infinite-layered infinite Cartesian grid. It is not known whether one cop can capture the robber on this graph. We have shown in this thesis that two cops suffice. One would want to prove that either one cop cannot capture the robber or if one cop suffices, then determine how many moves would be needed to capture the robber.

(3) $W_{c_{t}}\left(P_{\infty} \otimes P_{\infty} \otimes P_{\infty}\right)$.

This graph is the infinite-layered infinite strong grid. It is not known whether one cop can capture the robber on this graph. We have shown in this thesis that 14 cops suffice. One would want to prove that either one cop cannot capture the robber or if one cop suffices, then determine how many moves would be needed to capture the robber.

(4) $W_{c_{t}}$ of other graph products.

In this thesis, we have studied the Cartesian and strong graph products. There are 256 possible products, and some of the notable ones that we have not studied are the lexicographic, disjunction, and symmetric difference products [4]. 
(5) Wall robber capture time and wall robber cop number for trees and hypercubes.

All the graph classes we studied for the original game may also be studied for Wall Cops and Wall Robbers. It would be interesting to determine the wall robber capture time and wall robber cop number for trees and hypercubes. 


\section{Bibliography}

[1] B. Alspach, Sweeping and searching in graphs: a brief survey, Matematiche 59 (2006) 5-37.

[2] E.R. Berlekamp, J.H. Conway, R.K. Guy, Winning Ways for your Mathematical Plays, vol. 2, Academic Press, New York, 1982.

[3] A. Bonato, WHAT IS ... COP NUMBER? Notices of the American Mathematical Society 59 (2012) 1100-1101.

[4] A. Bonato, R.J. Nowakowski, The Game of Cops and Robber on Graphs, American Mathematical Society, Providence, Rhode Island, 2011, 80-82.

[5] J.H. Conway, The Angel Problem, in: R. Nowakowski (editor) Games of No Chance, volume 29 of MSRI Publications, 1996, 3-12.

[6] S. Finbow, G. MacGillivray, The Firefighter problem: a survey of results, directions and questions, Australasian Journal of Combinatorics 43 (2009) 57-77.

[7] G. Hahn, Cops, robbers and graphs, Tatra Mathematical Publications 36 (2007) 163-176.

[8] B. Hartnell, Firefighter! An application of domination. Presentation at the 25th Manitoba Conference on Combinatorial Mathematics and Computing, University of Manitoba, Winnipeg, Canada, 1995.

[9] R.J. Nowakowski, P. Winkler, Vertex-to-vertex pursuit in a graph, Discrete Mathematics 43 (1983) 235-239.

[10] A. Quilliot, Jeux et pointes fixes sur les graphes, Thèse de 3ème cycle, Université de Paris VI, 1978, 131-145. 